\title{
Indecomposable Modules Over the Virasoro Lie Algebra and a Conjecture of V. Kac*
}

\author{
Christiane Martin and Alain Piard \\ Physique-Mathématique, Université de Bourgogne, B.P. 138, F-21004 Dijon Cedex France
}

Received July 16, 1990

\begin{abstract}
We consider a class of indecomposable modules over the Virasoro Lie algebra that we call bounded admissible modules. We get results concerning the center and the dimensions of the weight spaces. We prove that these modules always contain a submodule with one-dimensional weight spaces. From this follows the proof of a conjecture of V. Kac concerning the classification of simple admissible modules.
\end{abstract}

\section{Introduction}

The Virasoro algebra $\mathscr{V}$ is the universal central extension of the complex Lie algebra $W$ of polynomial vector fields on the circle [1]. $W$ is also the Lie algebra of the group of diffeomorphisms of the circle. The Virasoro algebra plays a fundamental role in two dimensional conformal quantum field theory [2-5]. Therefore, the unitarizable $\mathscr{V}$-modules with highest or lowest weight have been extensively studied by many authors [5-8]. For the same reason it was of interest to determine whether the Verma modules are irreducible or not irreducible [9]. We can also notice that indecomposable Verma $\mathscr{V}$-modules occur in two dimensional conformal degenerate quantum field theory [4]. Besides highest or lowest weight $\mathscr{V}$-modules, another class of $\mathscr{V}$-modules was exhaustively classified by Kaplansky-Santharoubane [10]. These are the indecomposable $\mathscr{V}$-modules, where $x_{0}$ the rotation generator acts semisimply with one-dimensional eigenspaces. Such $\mathscr{V}$-modules have been previously introduced by Feigin-Fuchs [11]. The existence of the last type of $\mathscr{V}$-modules permits to confirm that the result of Kostrikin [12] concerning the simple Z-graduate Lie algebras of Cartan type other than $W_{1}$ (the algebra of the derivations of the polynomial ring $\mathbb{C}[X]$ ) is indeed false for $W_{1}$ as $W_{1}$ is a subalgebra of $\mathscr{V}$. Thus Victor Kac [13] conjectured the following theorem:

* Preprint Université de Bourgogne-mai 1990 
Theorem. Let $\mathscr{A}$ be an irreducible $\mathscr{V}$-module satisfying

a) $x_{0}$ acts semisimply,

b) The eigenspaces of $x_{0}$ are finite dimensional.

Then either $\mathscr{A}$ is a highest or lowest weight $\mathscr{V}$-module or $\mathscr{A}$ has all its weight spaces of dimension less than or equal to one.

This theorem has been proved by Pressley-Chari [14] for the unitarizable irreducible $\mathscr{V}$-modules.

Many reasons incite to study indecomposable $\mathscr{V}$-modules and not only irreducible ones: the distinguished role of $\mathscr{V}$ among the infinite $Z$-graduate Lie algebras, its connexion with physics and the emergence of indecomposable modules particularly in gauge theories.

In this paper, we are interested in indecomposable $\mathscr{V}$-modules satisfying the above conditions a) and b) and the additional following condition:

c) The dimensions of the eigenspaces of $x_{0}$ are uniformly bounded.

We give here complete proofs of results announced in [15]. The additional hypothesis c) clearly sets up a natural generalization of the class of $\mathscr{V}$-modules classified by Kaplansky and Santharoubane [10]. All extensions of these $\mathscr{V}$ modules by- themselves are actually indecomposable $\mathscr{V}$-modules satisfying our hypotheses. More, all these extensions appear to be indecomposable representations in which the eigenspaces of $x_{0}$ have a dimension less than or equal to two. And our methods enable us to give an explicit construction of these $\mathscr{V}$-modules. We shall draw up this construction in a forthcoming paper.

Our main result is the following: any $\mathscr{V}$-module satisfying a) b) c) contains a submodule where all the eigenspaces of $x_{0}$ have a dimension less than or equal to 1 . Thus we get the proof of the conjecture of V. Kac for simple $\mathscr{V}$-modules satisfying a), b) and c). Actually it gives the proof of the conjecture in the general case in view of the lemma (2.7) in [16]. In this preprint, $O$. Mathieu also gives a completely different proof of the conjecture in the general case. His method involves representations of finite dimensional restricted Lie algebras on finite fields. He does not give results concerning indecomposable $\mathscr{V}$-modules.

Let us give now a brief survey of this paper. We recall that the Virasoro algebra is the complex Lie algebra with basis $\left\{c, x_{n}, n \in Z\right\}$ and commutation relations:

$$
\left[x_{i} x_{j}\right]=(j-i) x_{i+j}+\delta_{i,-j} \frac{j^{3}-j}{12} c, \quad\left[c, x_{i}\right]=0 \quad \forall i \forall j \in Z .
$$

We consider an indecomposable $\mathscr{V}$-module satisfying the properties a), b) and c). The basic idea is to give a minimal condition on a given $\mathscr{V}$-module implying the existence of a submodule with one-dimensional weight spaces (eigenspaces of $x_{0}$ ). Then we show that this condition is generally verified in most of the cases. This minimal condition is the hypothesis of Theorem (II.10): there exists a common eigenvector $v$ of both $x_{0}$ and the Casimir $Q_{1}$ of the sl(2)-algebra generated by $\left\{x_{0}, x_{-1}, x_{1}\right\}$ such that $\left\{x_{1}^{2} v, x_{2} v\right\}$ is a dependent system. First we prove (Theorem (II.10)) that the $\mathscr{V}$-submodule generated by such a vector $v$ has one-dimensional weight spaces for enough high weights. This result and the fact that the only eigenvalue of the central element $c$ is zero are the essential points of Sect. II. In the case where $\operatorname{Ker} x_{-1}+\operatorname{Ker} x_{1}=0$, it is quite clear that the $\mathscr{V}$-submodule 
generated by $v$ has all its weight spaces of dimension one. In the case where $\operatorname{Ker} x_{-1}+\operatorname{Ker} x_{1} \neq 0$ we clearly need a careful study of the dimensions of weight spaces. This is the object of Sect. III. It can be easily seen (Sect. I) that the dimensions of weight spaces are constant for enough high or enough low weights. By Theorem (III.2) these two constants, called asymptotic dimensions of the $\mathscr{V}$-module, are shown to be equal. Then we prove more precisely that all the weight spaces have the same dimension maybe except one which is necessarily of zero weight. We also prove that the corresponding $\mathscr{V}$-module can never be simple when the zero weight space has a dimension higher than the dimension of all other weight spaces. These results are given by Theorem (III.5). From (III.5) and (II.10) follows immediately the existence of a $\mathscr{V}$-submodule with one-dimensional weight spaces for $\mathscr{V}$-modules such that $\operatorname{Ker} x_{-1}+\operatorname{Ker} x_{1} \neq\{0\}$. Then, the aim of Sect. IV is to find in most cases a vector $v$ satisfying the hypothesis of Theorem (II.10) in $\mathscr{V}$-modules such that $\operatorname{Ker} x_{-1}+\operatorname{Ker} x_{1}=\{0\}$. First we give necessary conditions for a representation where $\operatorname{Ker} x_{-1}+\operatorname{Ker} x_{1}=\{0\}$ to be indecomposable (Proposition (IV.5)) or irreducible (Theorem (IV.6)). Up to this point, we only need inducing arguments relative to generating commutation relations and an asymptotic analysis of the relation $\left[x_{-2} x_{+2}\right]=4 x_{0}+\frac{1}{2} c$ on a "good" basis. But here an exact analysis of this last relation is necessary to get more precise information. As this relation appears to be a rational expression of the integer $n$ indexing the weight spaces, we may consider some well chosen residue. Thus we get new relations which permit us either to get directly a proper $\mathscr{V}$-submodule or to obtain a vector $v$ satisfying the conditions of Theorem (II.10). In all cases the conjecture of $V$. Kac is proved. Finally, all indecomposable $\mathscr{V}$-modules satisfying a), b) and c) are finite length extensions of such simple $\mathscr{V}$-modules with one-dimensional weight spaces.

\section{Basic Properties}

\section{Notations - Definitions}

We recall that the Virasoro algebra $\mathscr{V}$ is the complex Lie algebra with basis $\left\{c, x_{n}, n \in Z\right\}$ and commutation relations:

$$
\begin{aligned}
{\left[x_{i}, x_{j}\right] } & =(j-i) x_{i+j}+\frac{j^{3}-j}{12} \delta_{i,-j} c, \\
{\left[c, x_{i}\right] } & =0 .
\end{aligned}
$$

We also recall that $\left\{x_{0}, x_{1}, x_{-1}, x_{2}, x_{-2}, c\right\}$ is a finite system of Lie generators of $\mathscr{V}$.

We call $\{x, y, h\}$ a standard basis of $\operatorname{sl}(2)$ when it satisfies the commutation relations:

$$
[x, y]=h, \quad[h, x]=2 x, \quad[h, y]=-2 y .
$$

We denote $\mathscr{G}_{i}$ the subalgebra of $\mathscr{V}$ isomorphic to $\operatorname{sl}(2)$ with standard basis

$$
\left\{\frac{x_{i}}{i},-\frac{x_{-i}}{i}, \frac{1}{i}\left(2 x_{0}+\frac{i^{2}-1}{12} c\right)\right\} \text {. }
$$

Its Casimir operator $Q_{i}$ is given by:

$$
i^{2} Q_{i}=-x_{i} x_{-i}+\left(x_{0}+\frac{i^{2}-1}{24} c\right)^{2}-i\left(x_{0}+\frac{i^{2}-1}{24} c\right) \text {. }
$$


Finally, we denote $\mathscr{V}_{i}$ the subalgebra of $\mathscr{V}$ isomorphic to $\mathscr{V}$ generated by $\left\{c, x_{k i}, k \in Z\right\}$.

Let us introduce the following definitions:

Definition (I.1.1). $A \mathscr{V}$-module $\mathscr{A}$ is said to be admissible if:

$-x_{0}$ acts semisimply on $\mathscr{A}$ and

- the eigenspaces of $x_{0}$ are finite-dimensional.

The eigenspaces of $x_{0}$ will be also called the weight spaces of $\mathscr{A}$.

Definition (I.1.2). An admissible $\mathscr{V}$-module $\mathscr{A}$ is said to be bounded if the dimensions of weight spaces are uniformly bounded.

Definition (I.1.3). $A \mathscr{V}$-module $\mathscr{A}$ is indecomposable if a decomposition of $\mathscr{A}$ in a direct sum of non-trivial $\mathscr{V}$-submodules does not exist.

Proposition (1.1.4). Let $\mathscr{A}$ be an admissible indecomposable $\mathscr{V}$-module. Then, there exists a decomposition $\mathscr{A}=\bigoplus_{n \in Z} \mathscr{A}_{a+n}$ such that $x_{0}(v)=(a+n) v \forall v \in \mathscr{A}_{a+n}$, where a is a fixed complex number which is unique when the condition $0 \leqq \operatorname{Re} a<1$ is imposed and $\mathscr{A}_{a+n}$ can be eventually trivial.

This is a direct consequence of the commutation relations $\left[x_{0}, x_{i}\right]=i x_{i}$ which imply $x_{i}\left(\mathscr{A}_{a+n}\right) \subset \mathscr{A}_{a+n+i}$.

Remark (I.1.5). For a non-trivial bounded admissible indecomposable $\mathscr{V}$-module, we shall see in part III that the only subspace $\mathscr{A}_{a+n}$ which may be $\{0\}$ is such that $a+n=0$.

\section{Standard Basis for a Bounded Admissible Indecomposable $\mathscr{V}$-Module}

Let $\mathscr{A}$ be such a $\mathscr{V}$-module. By Proposition (I.1.4) we have a decomposition $\mathscr{A}=\bigoplus_{n \in Z} \mathscr{A}_{a+n}$, where $\mathscr{A}_{a+n}$ are finite dimensional invariant by $Q_{1}$ with bounded dimensions. Thus, each subspace $\mathscr{A}_{a+n}$ can be decomposed into the direct sum of characteristic subspaces of $Q_{1}$. From this, we deduce a decomposition $\mathscr{A}=\oplus N_{i}$ into characteristic subspaces of $Q_{1}$ which are invariant by $\mathscr{G}_{1}$.

Lemma (I.2.1). On each characteristic subspace $N_{i}=\bigoplus_{n \in Z} N_{i} \cap \mathscr{A}_{a+n}$ there exists at most four subspaces $N_{i} \cap \mathscr{A}_{a+n}$ on which $x_{1}$ or $x_{-1}$ has a non-trivial kernel.

Indeed, a vector $v$ such that $x_{1} v=0$ or $x_{-1} v=0$ is an eigenvector of $Q_{1}$ so that we get:

$$
\begin{array}{ll}
Q_{1} v=\lambda_{i}\left(\lambda_{i}-1\right) v=(a+n+1)(a+n) v & \text { if } x_{i} v=0, \\
Q_{1} v=\lambda_{i}\left(\lambda_{i}-1\right) v=(a+n-1)(a+n) v & \text { if } x_{-i} v=0 .
\end{array}
$$

Each of these equations furnishes at most two solutions for the integer $n$.

Proposition (I.2.2). Let $\mathscr{A}=\bigoplus_{n \in Z} \mathscr{A}_{a+n}$ be a bounded admissible indecomposable $\mathscr{V}$-module. Then, there exists at most a finite number of weight spaces $\mathscr{A}_{a+n}$ on which $x_{1}$ or $x_{-1}$ has a non-trivial kernel.

Proof. If there exists an infinite number of characteristic subspaces $N_{i}$, we can suppose that an infinite number of them is intersecting the subspace $\bigoplus_{n \geqq n_{0}} \mathscr{A}_{a+n}$ where 
$\operatorname{Re}\left(a+n_{0}\right)>0$. Among them, as $\left\{N_{i} / N_{i} \cap \mathscr{A}_{a+n_{0}} \neq\{0\}\right\}$ is a finite set, there exists an infinite number of $N_{i}$ such that $x_{-1}$ has a non-trivial kernel in $N_{i} \cap \bigoplus_{n \geq n_{0}} \mathscr{A}_{a+n}$. Setting $q=\sup \left\{\operatorname{dim} \mathscr{A}_{a+n}, n \in Z\right\}$, there exists $q+1$ integers $n_{1} \leqq n_{2} \leqq \cdots \leqq n_{q+1}$ and $q+1$ vectors $\left\{v_{1} \cdots v_{q+1}\right\}$ such that $v_{l} \in \mathscr{A}_{a+n_{l}} \cap N_{i_{l}}$ and $x_{-1} v_{l}=0, l=1,2, \ldots, q+1$. The vectors $v_{l}$ are eigenvectors of $Q_{1}$ with different eigenvalues so that the set $\left\{v_{1}, \ldots, v_{q+1}\right\}$ is independent. As $\operatorname{Re}\left(a+n_{0}\right)>0$, we deduce that the set $\left\{x_{1}^{n_{q+1}-n_{1}} v_{1}\right.$, $\left.x_{1}^{n_{q+1}-n_{2}} v_{2}, \ldots, v_{q+1}\right\}$ is independent in $\mathscr{A}_{a+n_{q+1}}$, a contradiction with the definition of $q$. Thus the characteristic subspaces $N_{i}$ are of a finite number and by Lemma (I.2.1) we deduce the proposition.

In the rest of this paper, $p$ will be the number of characteristic subspaces $N_{i}$. Proposition (I.2.2) implies that any bounded admissible indecomposable $\mathscr{V}$-module is such that:

$$
\begin{array}{lll}
\operatorname{dim} \mathscr{A}_{a+n}=q & \forall n \geqq n_{1} & n_{1} \in \mathbb{N}, \\
\operatorname{dim} \mathscr{A}_{a+n}=r & \forall n \leqq-n_{2} & n_{2} \in \mathbb{N} .
\end{array}
$$

Definition (I.2.3). $q$ and $r$ are said to be the asymptotic dimensions of the bounded admissible indecomposable $\mathscr{V}$-module $\mathscr{A}$.

From now on, we shall use the following definition (I.2.4) for integers $k$ and $k^{\prime}$ :

- If $x_{1}$ or $x_{-1}$ has a non-trivial kernel in $\mathscr{A}$,

$$
\begin{aligned}
& k=\operatorname{Inf}\left\{n \in Z \text { such that } \operatorname{Ker} x_{1} \cap \bigoplus_{i \geqq n} \mathscr{A}_{a+i}=\operatorname{Ker} x_{-1} \cap \bigoplus_{i \geqq n} \mathscr{A}_{a+i}=\{0\}\right\}, \\
& k^{\prime}=\operatorname{Sup}\left\{n \in Z \text { such that } \operatorname{Ker} x_{1} \cap \bigoplus_{i \leqq n} \mathscr{A}_{a+i}=\operatorname{Ker} x_{-1} \cap \bigoplus_{i \leqq n} \mathscr{A}_{a+i}=\{0\}\right\} .
\end{aligned}
$$

- If $\operatorname{Ker} x_{1}=\operatorname{Ker} x_{-1}=\{0\}: k=k^{\prime}=0$.

We can notice that $\operatorname{dim} \mathscr{A}_{a+n}=q \forall n \geqq k$ and $\operatorname{dim} \mathscr{A}_{a+n}=r \forall n \leqq k^{\prime}$.

Proposition (I.2.5). Let $\mathscr{A}$ be a bounded admissible indecomposable $\mathscr{V}$-module. Then, there exists a basis $\left\{v_{i, n}, 1 \leqq i \leqq q, n \geqq k\right\}$ of the subspace $\bigoplus_{n \geqq k} \mathscr{A}_{a+n}$ such that for any
$n \geqq k$ :

- the matrix of $x_{1}$ from $\mathscr{A}_{a+n}$ to $\mathscr{A}_{a+n+1}$ is $(a+n) I+\Lambda+\Delta(n)$

- the matrix of $x_{-1}$ from $\mathscr{A}_{a+n+1}$ to $\mathscr{A}_{a+n}$ is $(a+n+1) I-\Lambda$,

where $\Lambda$ is a diagonal matrix equal to $\lambda_{i} I$ when restricted to $N_{i} \cap \mathscr{A}_{a+n}$ and $(a+n+1-\Lambda) \Delta(n)=(a+k+1-\Lambda) \Delta(k)$ is the opposite of the nilpotent part of a Jordan matrix of $Q_{1}$ on $\mathscr{A}_{a+k}$.

Proof. Let $\left\{v_{i, k} 1 \leqq i \leqq q\right\}$ a Jordan basis of $Q_{1}$ in $\mathscr{A}_{a+k}=\bigoplus_{j=1}^{p}\left(N_{j} \cap \mathscr{A}_{a+k}\right)$. For any $n \geqq k, x_{-1}$ is one to one from $N_{j} \cap \mathscr{A}_{a+n+1}$ to $N_{j} \cap \mathscr{A}_{a+n}$ so that we can define a Jordan basis $\left\{v_{i, k+1} 1 \leqq i \leqq q\right\}$ of $Q_{1}$ on $\mathscr{A}_{a+k+1}$ such that:

$$
x_{-1} v_{i, k+1}=\left(a+k+1-\lambda_{j}\right) v_{i, k} \text { for } v_{i, k} \in N_{j} \cap \mathscr{A}_{a+k} .
$$

Then by induction we get a Jordan basis $\left\{v_{i, n} 1 \leqq i \leqq q\right\}$ of $Q_{1}$ on $\mathscr{A}_{a+n}$ for all $n \geqq k$. On this basis, the Jordan matrix of $Q_{1}$ on $\mathscr{A}_{a+n}$ is $\Lambda(\Lambda-1)+J$, where $J$ is a constant 
nilpotent matrix for all $n \geqq k$ and the matrix of $x_{-1}$ from $\mathscr{A}_{a+n+1}$ to $\mathscr{A}_{a+n}$ is $(a+n+1) I-\Lambda$. We define $q \times q$ matrices $\Delta(n)$ such that, with respect to the chosen basis, we have: $x_{1} \mid \widehat{\mathscr{A}_{a+n}}=(a+n) I+\Lambda+\Delta(n)$. Then the relation $Q_{1}=-x_{-1} x_{1}+$ $x_{0}^{2}+x_{0}$ gives us:

$$
[(a+n+1) I-\Lambda)] \Delta(n)=-J \quad \forall n \geqq k .
$$

3. Elementary Properties of the Central Element $c$ for an Admissible $\mathscr{V}$-Module

\section{Proposition (I.3.1).}

a) In an irreducible admissible $\mathscr{V}$-module, $c$ acts by a scalar.

b) In an indecomposable admissible $\mathscr{V}$-module, $c$ has at most one eigenvalue.

In both cases, $c$ and $x_{0}$ commutes so that $\mathscr{A}_{a+n}$ is invariant by $c$. Thus, the $\mathscr{V}$-module $\mathscr{A}$ can be decomposed into a direct, at most countable sum of characteristic subspaces of $c$, each of them being invariant by $\mathscr{V}$. In case of an indecomposable $\mathscr{V}$-module $\mathscr{A}, c$ has at most one characteristic subspace. In case of an irreducible $\mathscr{V}$-module $\mathscr{A}$, the eigenspace of $c$ is invariant by $\mathscr{V}$ and we get the first part of Proposition (I.3.1).

Proposition (1.3.2). Let $\mathscr{A}=\bigoplus_{n \in Z} \mathscr{A}_{a+n}$ be a bounded admissible indecomposable $\mathscr{V}$-module. Then the restriction $c(n)$ of $c$ on $\mathscr{A}_{a+n}$ is such that $c(n)=c(k) \forall n \geqq k$, where $k$ is defined by (I.2.4).

For such a $\mathscr{V}$-module, we can consider the basis defined by Proposition (I.2.5). On this basis, the commutation relation $\left[c, x_{-1}\right]=0$ applied to $\mathscr{A}_{a+n+1}$ leads to:

$$
c(n)(a+n+1-\Lambda)=(a+n+1-\Lambda) c(n+1) .
$$

As $c$ commutes with $Q_{1}, c(n)$ commutes with $(a+n+1-\Lambda)$ Id which is an invertible matrix for all $n \geqq k$ and we deduce the result (I.3.2).

Remark (I.3.3). Analogously to the basis defined by (I.2.5) we can define a basis on $\bigoplus_{n \leqq k^{\prime}} \mathscr{A}_{a+n}\left(k^{\prime}\right.$ defined by (I.2.4)) and we can show also that $c(n)=c\left(k^{\prime}\right) \forall n \leqq k^{\prime}$.

\section{General Theorems for Admissible $\mathscr{V}$-Modules}

This part is mainly devoted to the proof of Theorem (II.10). It gives conditions on a bounded admissible $\mathscr{V}$-module asserting the existence of a submodule having, one-side, one dimensional asymptotic weight spaces. This theorem will be the main tool in Parts III and IV to get the proof of the conjecture of Victor Kac. It is also of crucial importance to get the general properties of bounded admissible $\mathscr{V}$-modules that we establish in Part III. Another result in this part is to prove that $c=0$ for any bounded admissible irreducible $\mathscr{V}$-module.

Let $\mathscr{A}=\bigoplus_{n \in Z} \mathscr{A}_{a+n}$ be a bounded admissible indecomposable $\mathscr{V}$-module and $k$ the integer defined by (I.2.4), then:

Proposition (II.1). Let $\mathscr{A}$ be a bounded admissible indecomposable $\mathscr{V}$-module and 
$v \in \mathscr{A}_{a+n_{0}}, n_{0} \geqq k$, an eigenvector of both $Q_{1}$ and $c$ such that

a) $x_{2} v=\alpha x_{1}^{2} v \quad \alpha \in \mathbb{C}$,

b) $x_{-2} x_{1}^{2} v=\alpha^{\prime} v \quad \alpha^{\prime} \in \mathbb{C}$,

then the submodule generated by $v$ has one-dimensional weight spaces in $\mathscr{A}_{a+n}$ for all $n \geqq n_{0}$.

Proof. Let $\mathscr{A}^{\prime}$ be the submodule of $\mathscr{A}$ generated by $v$. By the Poincaré-BirkhoffWitt theorem, $\mathscr{A}^{\prime}$ is generated as a vector space by the vectors $x_{-n_{1}} x_{-n_{2}} \cdots x_{-n_{i}} x_{m_{1}} \cdots$ $x_{m_{j}} v$, where $n_{k}$ and $m_{l} \in \mathbb{N}$. We can even assume that $n_{k}=1$ or 2 and $m_{l}=1$ or 2 .

- First, we prove that $x_{2} x_{1}^{m} v \in \mathbb{C} \cdot x_{1}^{m+2} v$ by induction on $m$. By hypothesis a) the property is verified for $m=0$. We can notice that for all $m \geqq 0 x_{1}^{m} v$ is an eigenvector of $Q_{1}$ so that $x_{-1} x_{1}^{m+1} v \in \mathbb{C} \cdot x_{1}^{m} v$. As $x_{-1}$ is injective on $\mathscr{A}_{a+n} \forall n \geqq n_{0}$, we induce the proof by use of the commutation relation $\left[x_{-1} x_{2}\right]=3 x_{1}$ applied to the vector $x_{1}^{m+1} v$. Hence we deduce that $\mathscr{A}^{\prime}$ is generated by the vectors $x_{-n_{1}} x_{-n_{2}} \cdots x_{-n_{i}} x_{1}^{m} v$, where $n_{1}, n_{2}, \ldots, n_{i} \in\{1,2\}$ and $m \in \mathbb{N}$.

- It remains to prove that $x_{-n_{1}} x_{-n_{2}} \cdots x_{-n_{i}} x_{1}^{m} v \in \mathbb{C} \cdot x_{1}^{m-n_{1}-n_{2} \cdots-n_{i}} v$ for all $m$ such that $m-n_{1}-\dot{n_{2}} \cdots-n_{i} \geqq 0$. We have already shown that $x_{-1} x_{1}^{m} v \in \mathbb{C} \cdot x_{1}^{m-1} v$ for all $m \geqq 1$. Thus, we have only to prove that $x_{-2} x_{1}^{m} v \in \mathbb{C} \cdot x_{1}^{m-2} v$ for all $m \geqq 2$. We also prove this result by induction on $m$. By hypothesis b) we have $x_{-2} x_{1}^{2} v=\alpha^{\prime} v$. Then we induce the property by use of the commutation relation $\left[x_{-2} x_{1}\right]=3 x_{-1}$ applied to the vector $x_{1}^{m} v$. Thus we get Proposition (II.1).

Now, in the conditions of Proposition (II.1), we want to prove that the hypothesis b) is implied by the hypothesis a). For this, we need to generalize the asymptotic study of the commutation relation $\left[x_{-2} x_{2}\right]$ that V. Chari and A. Pressley use in the unitary case [14]. Let $\left\{v_{i, n} i=1, \ldots, q n \geqq k\right\}$ be a basis given by Proposition (I.2.5). We define $q \times q$ matrices $A(n)$ and $B(n+2)$ for all $n \geqq k$ such that, with respect to this basis, $(a+n+2 \Lambda)+A(n)$ is the matrix of $x_{2}$ on $\mathscr{A}_{a+n}$ and $(a+n+2-2 \Lambda)+B(n+2)$ is the matrix of $x_{-2}$ on $\mathscr{A}_{a+n+2}$. The diagonal matrices $(a+n+2 \Lambda)$ and $(a+n+2-2 \Lambda)$ are defined as in Proposition (I.2.5).

'The commutation relation $\left[x_{-1} x_{2}\right]=3 x_{1}$ applied to $\mathscr{A}_{a+n}$ gives:

$$
(a+n+2-\Lambda) A(n)-A(n-1)(a+n-\Lambda)=3 \Delta(n),
$$

where $\Delta(n)$ is given by Proposition (I.2.5).

This relation implies:

$$
A(n)=\frac{\Gamma(a+k+3-\Lambda)}{\Gamma(a+n+3-\Lambda)} A(k) \frac{\Gamma(a+n+1-\Lambda)}{\Gamma(a+k+1-\Lambda)}+\frac{3(n-k)(a+k+1-\Lambda)}{(a+n+2-\Lambda)(a+n+1-\Lambda)} \Delta(k) .
$$

The gamma functions here denote the obvious diagonal matrices. They are invertible and commute with $\Delta(k)$. As $x_{-1}$ does not annihilate on $\mathscr{A}_{a+n+1}$ for all $n \geqq k$, we have $-\left(a+n+1-\lambda_{i}\right) \notin \mathbb{N}$ for all $i \in\{1, \ldots, p\}$. Thus the definition of $\Gamma$ functions and the formula (II.2) are quite clear.

Similarly, the commutation relation $\left[x_{-2} x_{1}\right]=3 x_{-1}$ applied to $\mathscr{A}_{a+n}$ for $n \geqq k+2$ gives:

$$
B(n+1)(a+n+\Lambda+\Delta(n))-(a+n-2+\Lambda+\Delta(n-2)) B(n)=F(n),
$$


where

$$
F(n)=\frac{(a+k+1-\Lambda)(a+n+1-3 \Lambda)}{(a+n+1-\Lambda)(a+n-1-\Lambda)} \Delta(k) .
$$

Hence, we deduce

$$
\begin{aligned}
B(n+1)= & \prod_{i=k}^{n-2}(a+i+\Lambda+\Delta(i)) B(k+2) \prod_{i=k+2}^{n}(a+i+\Lambda+\Delta(i))^{-1} \\
& +\left[\sum_{i=k+2}^{n} F(i)(a+i-1+\Lambda+\Delta(i-1))\right] \\
& \cdot\left(a+n-1+\Lambda+\Delta(n-1)^{-1}(a+n+\Lambda+\Delta(n))^{-1} .\right.
\end{aligned}
$$

Using the decomposition:

$$
\begin{aligned}
& (a+i-1+\Lambda+\Delta(i-1)) F(i) \\
& =(a+k+1-\Lambda) \Delta(k)\left[1+\frac{2 \Lambda(\Lambda-1)}{a+i+1-\Lambda}-\frac{2 \Lambda(\Lambda-1)}{a+i-1-\Lambda}\right] \\
& \quad+(a+k+1-\Lambda)^{2} \Delta(k)^{2}\left[\frac{-\Lambda}{a+i+1-\Lambda}+\frac{2 \Lambda-1}{a+i-\Lambda}+\frac{1-\Lambda}{a+i-1-\Lambda}\right],
\end{aligned}
$$

we get the following expression of $B(n+1)$ for all $n \geqq k+2$ :

$$
\begin{aligned}
B(n+1)= & \frac{\Gamma(a+n-1+\Lambda)}{\Gamma(a+k+\Lambda)} \prod_{i=k}^{n-2}\left(1+\frac{\Delta(i)}{a+i+\Lambda}\right) B(k+2) \\
& \cdot \prod_{i=k+2}^{n}\left(1+\frac{\Delta(i)}{a+i+\Lambda}\right)^{-1} \frac{\Gamma(a+k+2+\Lambda)}{\Gamma(a+n+1+\Lambda)} \\
& +\left\{\left[n-k-1+\frac{2 \Lambda(\Lambda-1)}{a+n+1-\Lambda}+\frac{2 \Lambda(\Lambda-1)}{a+n-\Lambda}-\frac{2 \Lambda(\Lambda-1)}{a+k+2-\Lambda}-\frac{2 \Lambda(\Lambda-1)}{a+k+1-\Lambda}\right]\right. \\
& \cdot(a+k+1-\Lambda) \Delta(k) \\
& +\left[\frac{-\Lambda}{a+n+1-\Lambda}+\frac{\Lambda}{a+k+2-\Lambda}+\frac{\Lambda-1}{a+n-\Lambda}-\frac{\Lambda-1}{a+k+1-\Lambda}\right] \\
& \left.\cdot(a+k+1-\Lambda)^{2} \Delta(k)^{2}\right\}(a+n-1+\Lambda+\Delta(n-1))^{-1}(a+n+\Lambda+\Delta(n))^{-1} .
\end{aligned}
$$

For $n \geqq k+3$ we define unique $B_{0}(n)$ and $H(n)$ by the relations:

$$
\begin{aligned}
B(n)= & B_{0}(n)+H(n), \\
B_{0}(n)= & \frac{\Gamma(a+n-2+\Lambda)}{\Gamma(a+k+\Lambda)} \prod_{i=k}^{n-3}\left(1+\frac{\Delta(i)}{a+i+\Lambda}\right) B(k+2) \\
& \cdot \prod_{i=k+2}^{n-1}\left(1+\frac{\Delta(i)}{a+i+\Lambda}\right)^{-1} \frac{\Gamma(a+k+2+\Lambda)}{\Gamma(a+n+\Lambda)} .
\end{aligned}
$$

Then, the commutation relation $\left[x_{-2} x_{2}\right]=4 x_{0}+\frac{1}{2} c$ applied to $\mathscr{A}_{a+n}$ for $n \geqq k+3$ 
gives:

$$
\begin{aligned}
- & (a+n+2-2 \Lambda) A(n)+A(n-2)(a+n-2 \Lambda)-B(n+2)(a+n+2 \Lambda) \\
& +(a+n-2+2 \Lambda) B(n)=-\frac{1}{2} c(n)-A(n-2) B(n)+B(n+2) A(n) .
\end{aligned}
$$

Substituting from (II.2) and (II.4) into (II.5), multiplying on the left by $\frac{\Gamma(a+n+1-\Lambda)}{\Gamma(a+k+3-\Lambda)}$, on the right by $\frac{\Gamma(a+k+1-\Lambda)}{\Gamma(a+n+1-\Lambda)}$, we get an equation (E). To study the asymptotic behaviour of this equation, we need the following lemma $[14,17$ Sect. 12.33].

Lemma (II.6). For any $\mu, v \in \mathbb{C}$, we have $\lim _{n \rightarrow \infty} \frac{\Gamma(n+a+\mu) \Gamma(n+a-\mu)}{\Gamma(n+a+v) \Gamma(n+a-v)}=1$.

In view of this lemma, the terms in equation (E) are $O\left(1 / n^{2}\right)$ and $O(1)$. We get precisely:

$$
\begin{aligned}
& \cdot \frac{\Gamma(a+n+1-\Lambda)}{\Gamma(a+k+3-\Lambda)}[-(a+n+2-2 \Lambda) A(n)+A(n-2)(a+n-2 \Lambda)] \frac{\Gamma(a+k+1-\Lambda)}{\Gamma(a+n+1-\Lambda)} \\
& \quad \sim \frac{1}{n^{2}}\left[2 A(k)-6 \frac{(a+1+k) \Delta(k)}{a+k+2-\Lambda}\right] \\
& \cdot \frac{\Gamma(a+n+1-\Lambda)}{\Gamma(a+k+3-\Lambda)}\left[-B_{0}(n+2)(a+n+2 \Lambda)+(a+n-2+2 \Lambda) B_{0}(n)\right] \frac{\Gamma(a+k+1-\Lambda)}{\Gamma(a+n+1-\Lambda)} \\
& \quad \sim \frac{1}{n^{2}} \frac{2}{\Gamma(a+k+\Lambda) \Gamma(a+k+3-\Lambda)} \prod_{i=k}^{+\infty}\left(1+\frac{\Delta(i)}{a+i+\Lambda}\right) B(k+2) \\
& \quad \cdot \prod_{i=k+2}^{+\infty}\left(1+\frac{\Delta(i)}{a+i+\Lambda}\right)^{-1} \Gamma(a+k+2+\Lambda) \Gamma(a+k+1-\Lambda),
\end{aligned}
$$

where infinite products converge since $\frac{\Delta(i)}{a+i+\Lambda} \sim \frac{(a+k+1-\Lambda) \Delta(k)}{i^{2}}$,

$$
\begin{aligned}
& \cdot \frac{\Gamma(a+n+1-\Lambda)}{\Gamma(a+k+3-\Lambda)}[-H(n+2)(a+n+2 \Lambda)+(a+n-2+2 \Lambda) H(n)] \frac{\Gamma(a+k+1-\Lambda)}{\Gamma(a+n+1-\Lambda)} \\
& \sim \frac{2}{a+k+2-\Lambda} \frac{1}{n^{2}}\left[-\Delta(k)\left(a+k+1+\frac{2 \Lambda(\Lambda-1)}{a+k+2-\Lambda}+\frac{2 \Lambda(\Lambda-1)}{a+k+1-\Lambda}\right)\right. \\
& \left.\quad+(a+k+1-\Lambda) \Delta(k)^{2}\left(\frac{\Lambda}{a+k+2-\Lambda}-\frac{\Lambda-1}{a+k+1-\Lambda}\right)\right] \\
& \cdot \frac{\Gamma(a+n+1-\Lambda)}{\Gamma(a+k+3-\Lambda)} B(n+2) A(n) \frac{\Gamma(a+k+1-\Lambda)}{\Gamma(a+n+1-\Lambda)} \sim \frac{3}{n^{2}} \frac{a+k+1-\Lambda}{a+k+2-\Lambda} \Delta(k)^{2} \\
& \frac{\Gamma(a+n+1-\Lambda)}{\Gamma(a+k+3-\Lambda)} A(n-2) B(n) \frac{\Gamma(a+k+1-\Lambda)}{\Gamma(a+n+1-\Lambda)} \sim \frac{3}{n^{2}} \frac{a+k+1-\Lambda}{a+k+2-\Lambda} \Delta(k)^{2} \\
& \cdot-\frac{1}{2} \frac{\Gamma(a+n+1-\Lambda)}{\Gamma(a+k+3-\Lambda)} c(n) \frac{\Gamma(a+k+1-\Lambda)}{\Gamma(a+n+1-\Lambda)} \\
& \quad=-\frac{1}{2(a+k+2-\Lambda)(a+k+1-\Lambda)} c(k) \quad \forall n \geqq k .
\end{aligned}
$$


The last equality results from Proposition (I.3.2). Letting $n \rightarrow \infty$ in equation (E) therefore gives $c(n)=0 \forall n \geqq k$. In view of (I.3.1) and (I.3.3), we get:

\section{Theorem (II.7)}

1. In an irreducible bounded admissible $\mathscr{V}$-module $\mathscr{A}, c$ acts trivially.

2. In an indecomposable bounded admissible $\mathscr{V}$-module $\mathscr{A}, c$ has the only eigenvalue 0 . If $\mathscr{A}^{\prime}$ is the eigenspace of $c$ in $\mathscr{A}$, then $\mathscr{A} / \mathscr{A}^{\prime}$ is a finite dimensional $\mathscr{A}$-module.

Returning to equation (E), we multiply both sides by $n^{2}$ and let $n \rightarrow \infty$. This gives:

$$
\begin{aligned}
& \Gamma(a+k+3-\Lambda) \Gamma(a+k+\Lambda) A(k)+\prod_{i=k}^{+\infty}\left(1+\frac{\Delta(i)}{a+i+\Delta}\right) B(k+2) \\
& \quad \cdot \prod_{i=k+2}^{+\infty}\left(1+\frac{\Delta(i)}{a+i+\Lambda)}\right)^{-1} \Gamma(a+k+2+\Lambda) \Gamma(a+k+1-\Lambda) \\
& =\Gamma(a+k+\Lambda) \Gamma(a+k+2-\Lambda)\left[\left(4(a+k+1)+\frac{2 \Lambda(\Lambda-1)}{a+k+1-\Lambda}+\frac{2 \Lambda(\Lambda-1)}{a+k+2-\Lambda}\right) \Delta(k)\right. \\
& \left.\quad+\left(\frac{-\Lambda}{a+k+2-\Lambda}+\frac{\Lambda-1}{a+k+1-\Lambda}\right)(a+k+1-\Lambda) \Delta(k)^{2}\right] .
\end{aligned}
$$

From this equation, we deduce the following:

Proposition (II.9). Let $\mathscr{A}$ be an indecomposable bounded admissible $\mathscr{V}$-module. Let $v$ be an eigenvector of $Q_{1}$ in $\mathscr{A}_{a+n_{0}}, n_{0} \geqq k$ such that $x_{2} v=\alpha x_{1}^{2} v \alpha \in \mathbb{C}$. Then $c v=0$ and $x_{-2} x_{1}^{2} v=\alpha^{\prime} v \alpha^{\prime} \in \mathbb{C}$.

Proof. From (I.3.2) and (II.7) $v$ verifies $c v=0$. As $v$ is an eigenvector of $Q_{1}$, the basis of $\bigoplus_{n \geq k} \mathscr{A}_{a+n}$ given by Proposition (I.2.3) can be chosen so that $v$ is the first vector of the basis in $\mathscr{A}_{a+n_{0}}: v=v_{1, n_{0}}$. The hypothesis $x_{2} v=\alpha x_{1}^{2} v$ imply that all terms of the first column in $A\left(n_{0}\right)$ are zero maybe except the first one. The first column of $\Delta\left(n_{0}\right)$, and hence of $\Delta(k)$ is zero. Thus from (II.2), the same property is valid for the first column of $A(k)$. Moreover $\Delta(k)$ is a triangular nilpotent matrix. Thus the matrices $\prod_{i=l}^{+\infty}\left(1+\frac{\Delta(i)}{a+i+\Lambda}\right)$ are unipotent for any $l \in \mathbb{N}$. From (II.8), it results that all elements of the first column of $B(k+2)$ are zero except maybe the first one. From (II.3), we get the same property for $B\left(n_{0}+2\right)$. Finally, as $x_{1}^{2} v$ is a non-zero multiple of $v_{1, n_{0}+2}$, we get $x_{-2} x_{1}^{2} v=\alpha^{\prime} v$. From Proposition (II.1) and (II.9) we get the following:

Theorem (II.10). Let $\mathscr{A}=\bigoplus_{n \in Z} \mathscr{A}_{a+n}$ be an indecomposable bounded admissible $\mathscr{V}$-module. Let $v \in \mathscr{A}_{a+n_{0}}, n_{0} \geqq k$, be an eigenvector of $Q_{1}$ such that $x_{2} v=\alpha x_{1}^{2} v$. Then the $\mathscr{V}$-submodule of $\mathscr{A}$ generated by $v$ has one-dimensional weight spaces in $\mathscr{A}_{a+n}$ for all $n \geqq n_{0}$.

\section{Bounded Admissible $\mathscr{V}$-Modules where $\operatorname{Ker} x_{-1}+\operatorname{Ker} x_{1} \neq\{0\}$}

As one of the aims of this paper is the proof of the conjecture of V. Kac for bounded admissible $\mathscr{V}$-modules, in view of Theorem (II.10), the study of asymptotic 
dimensions of these $\mathscr{V}$-modules appears to be quite natural. We shall here precisely prove that asymptotic dimensions of indecomposable bounded admissible $\mathscr{V}$-modules are equal. As this result is evident when $\operatorname{Ker} x_{-1}+\operatorname{Ker} x_{1}=\{0\}$, we have only to prove it when $\operatorname{Ker} x_{-1}+\operatorname{Ker} x_{1} \neq\{0\}$. It will be the first step to get the proof of the conjecture for such $\mathscr{V}$-modules. First we need the following technical property:

Proposition (III.1). Let $\mathscr{A}$ be an indecomposable bounded admissible $\mathscr{V}$-module where $\operatorname{Ker} x_{-1} \neq\{0\}$. Let $m_{0}=\operatorname{Sup}\left\{n \in \mathbb{N} / \operatorname{Ker} x_{-1} \cap \mathscr{A}_{a+n} \neq\{0\}\right\}$. Let $v$ be a vector annihilated by $x_{-1}$ in $\mathscr{A}_{a+m_{0}}$. Assume $v$ verify one of the following conditions:

a) $x_{1}^{n} v \neq 0 \forall n \in \mathbb{N}$,

b) $\exists m_{1} \in \mathbb{N}$ such that $x_{1}^{m_{1}+1} v=0, x_{1}^{m_{1}} v \neq 0$ and $\exists v^{\prime} \in \mathscr{A}_{a+m_{1}+1}$ with $x_{-1} v^{\prime}=x_{1}^{m_{1}} v$.

Then the $\mathscr{V}$-submodule generated by $v$ has one-dimensional weight spaces for all $n \geqq k(k$ defined by (I.2.4)).

Proof. Let

$$
\begin{array}{lll}
w=x_{1}^{k-m_{0}} v & \text { if } x_{1}^{n} v \neq 0 \quad \forall n \in \mathbb{N} & \text { (case a)) } \\
w=x_{1}^{k-m_{1}-1} v^{\prime} & \text { if } x_{1}^{m_{1}+1} v=0 \quad \text { and } \quad x_{-1} v^{\prime}=x_{1}^{m_{1}} v & \text { (case b)). }
\end{array}
$$

We want to show that $w$ satisfies the hypothesis of Theorem (II.10). As $w$ is an eigenvector of $Q_{1}$, we have only to prove that $x_{2} w=\alpha x_{1}^{2} w \alpha \in \mathbb{C}$. First we get that for $n \geqq 2, x_{2} x_{1}^{n-2} v \in \mathbb{C} \cdot x_{1}^{n} v$ if $x_{1}^{n} v \neq 0$. Indeed, from the relation $\left[x_{2} x_{-1}\right]=-3 x_{1}$ we have $x_{-1} x_{2} v=3 x_{1} v$. If $x_{1}^{2} v \neq 0, x_{-1} x_{1}^{2} v$ is a non-zero multiple of $x_{1} v$. As $x_{-1}$ is injective on $\mathscr{A}_{a+m_{0}+2}$, we deduce that $x_{2} v \in \mathbb{C} \cdot x_{1}^{2} v$ if $x_{1}^{2} v \neq 0$. Then we induce the proof up to $n$ if $x_{1}^{n} v \neq 0$. Thus we get

- under hypothesis a): $x_{2} x_{1}^{k-m_{0}} v \in \mathbb{C} \cdot x_{1}^{k-m_{0}+2} v$ that is $x_{2} w \in \mathbb{C} \cdot x_{1}^{2} w$

- under hypothesis b): If $m_{1} \geqq 2, x_{2} x_{1}^{m_{1}-2} v \in \mathbb{C} \cdot x_{1}^{m_{1}} v$. In this case, we apply the relation $\left[x_{-1} x_{2}\right]=3 x_{1}$ to $x_{1}^{m_{1}-1} v, x_{1}^{m_{1}} v$ and $v^{\prime}$. Using the injectivity of $x_{-1}$ on $\mathscr{A}_{a+m_{0}+m_{1}+i}(i=1,2,3)$ and the definition of $v^{\prime}$, we get: $x_{2} x_{1}^{m_{1}-1} v \in \mathbb{C} \cdot v^{\prime}, x_{2} x_{1}^{m_{1}} v \in$ $\mathbb{C} \cdot x_{1} v^{\prime}, x_{2} v^{\prime} \in \mathbb{C} \cdot x_{1}^{2} v^{\prime}$

By induction on $n$, then we get $x_{2} x_{1}^{n} v^{\prime} \in \mathbb{C} \cdot x_{1}^{n+2} v^{\prime}$ for all $n \in \mathbb{N}$. For $n=k-m_{1}-1$, this gives $x_{2} w=\alpha x_{1}^{2} w \alpha \in \mathbb{C}$.

If $m_{1}=0$ or $m_{1}=1$, first applying the relation $\left[x_{-1} x_{2}\right]=3 x_{1}$ to $v$ and then iterating, the proof is similar.

We can now prove the following theorem:

Theorem (III.2). The asymptotic dimensions $(q, r)$ of an indecomposable bounded admissible $\mathscr{V}$-module are equal.

Proof. This result is clear when $\operatorname{Ker} x_{-1}+\operatorname{Ker} x_{1}=\{0\}$. Thus let us assume $\operatorname{Ker} x_{-1}+\operatorname{Ker} x_{1} \neq\{0\}$ and suppose that $q \neq r$. As we can consider the inverse module, notation introduced in .[11], we may suppose $q>r$. If necessary, we can also consider a subalgebra $\mathscr{V}_{i}$ and a $\mathscr{V}_{i}$-submodule for enough large $i$ so that we may suppose:

$$
\operatorname{dim} \mathscr{A}_{a+n}=q \quad \forall n \geqq 0, \quad \operatorname{dim} \mathscr{A}_{a+n}=r \quad \forall n<0 \quad \text { and } \quad \operatorname{Re} a>0 .
$$

Now we shall get a contradiction by induction on $q$. 
- If $q=1, r=0$ : as $\operatorname{Re} a>0$, applying (I.2.5), we can choose a basis $\left\{v_{n}, n \in \mathbb{N}\right\}$ of $\mathscr{A}=\bigoplus_{n \in \mathbb{N}} \mathscr{A}_{a+n}$ such that:

$$
\begin{aligned}
x_{1} v_{n} & =(2 a+n) v_{n+1} & & \forall n \in \mathbb{N}, \\
x_{-1} v_{n} & =n v_{n-1} & & \forall n \in \mathbb{N} .
\end{aligned}
$$

From the relation $\left[x_{-1} x_{2}\right]=3 x_{1}$, we get by induction:

$$
x_{2} v_{n}=(3 a+n) v_{n+2} \text {. }
$$

As $x_{-2} v_{0}=x_{-2} v_{1}=0$, from the relation $\left[x_{1} x_{-2}\right]=-3 x_{-1}$ we deduce

$$
x_{-2} v_{n}=\frac{n(n-1)(3 a+n-2)}{(2 a+n-2)(2 a+n-1)} v_{n-2} \quad \forall n \in \mathbb{N} .
$$

Then the relation $\left[x_{2} x_{-2}\right]\left(v_{n}\right)=-4 x_{0} v_{n}(c=0$ in view of (II.7)) leads to a contradiction.

Suppose now that the case $q=n, r<n$ is impossible.

- If $q=n+1$ and $r<n+1, \operatorname{Ker} x_{-1} \cap \mathscr{A}_{a}$ is not trivial. We define $m_{0}$ as in (III.1). There exists $v$ annihilated by $x_{-1}$ in $\mathscr{A}_{a+m_{0}}$. As $\operatorname{Re} a>0$ we have $x_{1}^{n} v \neq 0 \forall n \in \mathbb{N}$. From Proposition (III.1), there exists in $\mathscr{A}$ a $\mathscr{V}$-submodule $\mathscr{A}^{\prime}$ with one-dimensional weight spaces in $\mathscr{A}_{a+n}$ for all $n \geqq k$. The asymptotic dimensions of $\mathscr{A}^{\prime}$ are $\left(1, r^{\prime}\right)$ with $1 \leqq r^{\prime} \leqq r$. The asymptotic dimensions of the quotient module $\mathscr{A} / \mathscr{A}^{\prime}$ are $\left(n, r-r^{\prime}\right)$ with $r-r^{\prime}<n$. Considering a suitable subalgebra $\mathscr{V}_{i}$ and applying the inductive hypothesis to a $\mathscr{V}_{i}$-submodule over $\mathscr{A} / \mathscr{A}^{\prime}$, we get a contradiction.

Corollary (III.3). All bounded admissible $\mathscr{V}$-modules with highest or lowest weight are finite direct sums of trivial $\mathscr{V}$-modules.

Definition (III.4). The integer $q$ such that $\operatorname{dim} \mathscr{A}_{a+n}=q \forall n \geqq k$ and $\forall n \leqq k^{\prime}$ is said to be the asymptotic dimension of the bounded admissible $\mathscr{V}$-module $\mathscr{A}$.

Now we shall improve the result of Theorem (III.2). Precisely, we want to show that all the weight spaces of an indecomposable bounded admissible $\mathscr{V}$-module have the same dimension maybe except one which is necessarily of zero-weight.

First we prove this result for the asymptotic dimension $q=1$.

Theorem (III.5). Let $\mathscr{A}$ be an indecomposable bounded admissible $\mathscr{A}$-module with asymptotic dimension one. Then all the weight spaces are one-dimensional except maybe the zero-weight space. Moreover, we have $\operatorname{dim} \mathscr{A}_{a+n} \leqq 1 \forall n \in Z$, if $\mathscr{A}$ is an irreducible $\mathscr{V}$-module.

We need the two following lemmas.

Lemma (III.6). Let $\mathscr{A}=\bigoplus_{n \in Z} \mathscr{A}_{a+n}$ be a bounded admissible $\mathscr{V}$-module such that $\operatorname{dim} \mathscr{A}_{a+n}=1 \forall n \in Z^{*}$ and $\operatorname{dim} \mathscr{A}_{a}>1$. Then $a=0$.

Proof. Setting $n_{0}=\operatorname{dim} \mathscr{A}_{a}$, we have $\operatorname{dim}\left(\operatorname{Ker} x_{-1} \cap \mathscr{A}_{a}\right)=n_{0}-1$ and $\operatorname{dim}\left(\operatorname{Ker} x_{1} \cap \mathscr{A}_{a}\right)=n_{0}-1$. If $n_{0} \geqq 3$ there exists obviously $v_{0} \in \mathscr{A}_{a}$ annihilated by $x_{-1}$ and $x_{1}$. This gives $a=0$. For $n_{0}=2$ two cases may occur:

- either there exists $v_{0} \in \operatorname{Ker} x_{-1} \cap \operatorname{Ker} x_{1} \cap \mathscr{A}_{0}$, hence $a=0$.

- or there exists a basis $\left\{v_{0}, v^{\prime}{ }_{0}\right\}$ of $\mathscr{A}_{0}$ such that $x_{-1} v_{0}=x_{1} v_{0}^{\prime}=0$. 
This gives

$$
\begin{aligned}
& Q_{1} v_{0}=\left(a^{2}-a\right) v_{0}, \\
& Q_{1} v_{0}^{\prime}=\left(a^{2}+a\right) v_{0}^{\prime} .
\end{aligned}
$$

Assume $a \neq 0$. As we can consider the inverse module [11] we may suppose $\operatorname{Re} a>0$. Then for all $n \in \mathbb{N}, x_{1}^{n} v_{0}$ is non-zero. By Proposition (I.2.5), we can choose a basis $\left\{v_{n}, n \in \mathbb{N}^{*}\right\}$ of $\bigoplus_{n \in \mathbf{N}^{*}} \mathscr{A}_{a+n}$ such that:

$$
\begin{aligned}
x_{0} v_{n} & =(a+n) v_{n}, \\
x_{-1} v_{n} & =n v_{n-1} \quad \forall n \in \mathbb{N}, \\
x_{1} v_{n} & =(2 a+n) v_{n+1} .
\end{aligned}
$$

From the relation $\left[x_{-1} x_{2}\right]=3 x_{1}$ we deduce:

$$
x_{2} v_{n}=(3 a+n) v_{n+2} \quad \forall n \in \mathbb{N} \text {. }
$$

Writing (II.2) for $k=1$, we get $A(1)=0$. Then (II.8) and (II.4) gives:

$$
x_{-2} v_{n}=(n-a) v_{n-2} \quad \forall n \geqq 3 .
$$

The relation $\left[x_{-2} x_{1}\right] v_{2}=3 x_{-1} v_{2}$ gives:

$$
x_{-2} v_{2}=(2-a) v_{0}+\mu v_{0}^{\prime} .
$$

Hence from the relation $\left[x_{-2} x_{2}\right] v_{2}=4 x_{0} v_{2}$ we get $\mu x_{2} v_{0}^{\prime}=0$. As $a \neq 0,\left\{v_{0}^{\prime}\right\}$ cannot be the trivial $\mathscr{V}$-module and $x_{2} v_{0}^{\prime} \neq 0$. Thus we have $\mu=0$. The relation $\left[x_{-2} x_{1}\right] v_{1}=3 x_{-1} v_{1}$ gives $x_{1} x_{-2} v_{1}=(a-1)(1-2 a) v_{0} .\left\{v_{0}^{\prime}\right\}$ and $\mathscr{A}_{-1}$ are eigenspaces of $Q_{1}$ with the same eigenvalue. Hence we have $x_{1} x_{-2} v_{1} \in \mathbb{C} \cdot v_{0}^{\prime}$ and we get: $x_{1} x_{-2} v_{1}=0$. If $x_{-2} v_{1} \neq 0, x_{1}$ annihilates $\mathscr{A}_{-1}$. Then $\bigoplus_{i \leqq-1} \mathscr{A}_{i}$ is a $\mathscr{G}_{1}$-submodule of the $\mathscr{G}_{1}$-module generated by $v_{0}^{\prime}$. Thus the corresponding $\mathscr{G}_{1}$-quotient module would be trivial. An this is impossible since $a \neq 0$. If $x_{-2} v_{1}=0$, the relation $\left[x_{-2} x_{1}\right] v_{0}=0$ gives $x_{1} x_{-2} v=0$. As $a \neq 0$, in view of (III.3), the case $x_{-2} v_{0}=0$ is impossible. Thus $x_{1}$ annihilates $\mathscr{A}_{-2}$. From the relation $\left[x_{1} x_{-2}\right] v_{0}^{\prime}=-3 x_{-1} v_{0}^{\prime}$ we get $x_{-1} v_{0}^{\prime}=0$. And this is impossible since $a \neq 0$. Therefore the assumption $a \neq 0$ is rejected.

Lemma (III.7). Let $\mathscr{A}=\bigoplus_{n \in Z} \mathscr{A}_{a+n}$ be a bounded admissible $\mathscr{V}$-module such that $\operatorname{dim} \mathscr{A}_{a+n}=1 \forall n \in Z^{*}$ and $\operatorname{dim} \mathscr{A}_{a}=0$. Then $a=0$.

Proof. $x_{-1}$ annihilates $\mathscr{A}_{a+1}$ and $x_{1}$ annihilates $\mathscr{A}_{a-1}$. Let us first show that they don't annihilate elsewhere.

Assume $x_{1}$ annihilates $\mathscr{A}_{a+n_{0}} n_{0} \neq-1$. If $n_{0}>0$, then $\bigoplus_{i=1}^{n_{0}} \mathscr{A}_{a+i}$ is a finite dimensional $\mathscr{G}_{1}$-module. This implies $a+1 \leqq 0$. From the relation $\left[x_{1} x_{n_{0}+1}\right]=n_{0} x_{n_{0}+2}$ applied to $\mathscr{A}_{a-1}$, we deduce that $x_{n_{0}+2}$ annihilates $\mathscr{A}_{a-1}$. Thus all $x_{i}, i \geqq n_{0}+2$, annihilates $\mathscr{A}_{a-1}$. Considering the Virasoro subalgebra $\mathscr{V}_{n_{0}+2}$ and applying Corollary (III.3), we' get $a-1=0$. An this gives a contradiction. If $n_{0}<-1$, from the same relation we deduce that $x_{-1}$ annihilates $\mathscr{A}_{a-1}$ so that $a-1=0$. Applying the relation $\left[x_{-1} x_{-2}\right]=-x_{-3}$ to $\mathscr{A}_{a+1}$, we prove that $x_{-3}$ annihilates $\mathscr{A}_{a+1}$. Hence all $x_{-i}$, 
$i \geqq 3$, annihilate $\mathscr{A}_{a+1}$. Thus we get $a+1=0$. And this is also a contradiction. Therefore $x_{1}$ only annihilates $\mathscr{A}_{a-1}$. Similarly $x_{-1}$ only annihilates $\mathscr{A}_{a+1}$. that:

From Proposition (I.2.5), there exists a basis $\left\{v_{n}, n \in \mathbb{N}^{*}\right\}$ of $\bigoplus_{n \in \mathbb{N}^{*}} \mathscr{A}_{a+n}$ such

$$
\begin{aligned}
x_{0} v_{n} & =(a+n) v_{n}, \\
x_{1} v_{n} & =(2 a+n+1) v_{n+1} \quad \forall n \in \mathbb{N}^{*}, \\
x_{-1} v_{n} & =(n-1) v_{n-1} .
\end{aligned}
$$

From the relation $\left[x_{-1} x_{2}\right]=3 x_{1}$ we get:

$$
x_{2} v_{n}=(3 a+n+2) v_{n+2} \quad \forall n \geqq 1 \text {. }
$$

Considering the formulas (II.2) for $k=2$, we deduce $A(2)=0$. Then (II.8) and (II.4) gives:

$$
x_{-2} v_{n}=(n-a-2) v_{n-2} \quad \forall n \geqq 4 .
$$

As $x_{-2} v_{2}=0$ the relation $\left[x_{1} x_{-2}\right]=-3 x_{1}$ applied to $v_{3}$ and $v_{2}$ gives:

$$
a(2 a+1)=0 \text {. }
$$

For $a=-\frac{1}{2}$, we have $\left[x_{2} x_{-2}\right] v_{2} \neq-4 x_{0} v_{2}$ so that necessarily $a=0$.

Proof of Theorem (III.5): Let $\mathscr{A}$ be an indecomposable bounded admissible $\mathscr{V}$-module with asymptotic dimension one. Assume there exists one weight space at least with a dimension different from one. We set:

$$
\begin{aligned}
& n_{1}=\sup \left\{n / \operatorname{dim} \mathscr{A}_{a+n} \neq 1\right\}, \\
& n_{2}=\operatorname{Inf}\left\{n / \operatorname{dim} \mathscr{A}_{a+n} \neq 1\right\} .
\end{aligned}
$$

Then $\bigoplus_{i \in Z} \mathscr{A}_{a+n_{1}+i\left(n_{1}-n_{2}+1\right)}$ and $\bigoplus_{i \in Z} \mathscr{A}_{a+n_{2}+i\left(n_{1}-n_{2}+1\right)}$ are modules over the Virasoro subalgebra $\mathscr{V}_{n_{1}-n_{2}+1}$. These modules satisfy the assumptions of Lemmas (III.6) and (III.7). Thus we deduce $a+n_{1}=a+n_{2}=0, n_{1}=n_{2}$. The first assertion of the theorem is obtained by the preceding lemmas.

It remains to show that $\mathscr{A}$ cannot be irreducible when $\mathscr{A}=\bigoplus_{i \in Z} \mathscr{A}_{i}$ and $\operatorname{dim} \mathscr{A}_{a} \geqq 2$. Let $\left\{v_{i}, i \in Z^{*}\right\}$ be a basis of $\bigoplus_{i \in Z^{*}} \mathscr{A}_{i}$.

If $x_{1} v_{1}$ and $x_{-1} v_{-1}$ are both non-zero, from the relations $\left[x_{-2} x_{1}\right] v_{1}=3 x_{-1} v_{1}$ and $\left[x_{2} x_{-1}\right] v_{-1}=-3 x_{1} v_{-1}$ we deduce that the subspace spanned by $\left\{x_{-1} v_{1}\right.$, $\left.x_{1} v_{-1}, v_{i} i \in Z^{*}\right\}$ is a $\mathscr{V}$-submodule. In the case where $\mathscr{A}_{0}$ is not spanned by $\left\{x_{-1} v_{1}, x_{1} v_{-1}\right\}$, then $\mathscr{A}$ is not irreducible. Suppose now that $\left\{x_{-1} v_{1}, x_{1} v_{-1}\right\}$ is a basis of $\mathscr{A}_{0}$. We set $v_{0}=x_{-1} v_{1}, v_{0}^{\prime}=x_{1} v_{-1}$. We have $x_{1} x_{-1} v_{0}=x_{-1} x_{1} v_{0}=0$. This implies $x_{1} v_{0}=x_{-1} v_{0}=0$ and similarly $x_{1} v^{\prime}{ }_{0}=x_{-1} v_{0}^{\prime}=0$. From the relation $\left[x_{1} x_{-2}\right] v_{0}=0$, we get $x_{1} x_{-2} v_{0}=0$. Assuming $x_{-2} v_{0} \neq 0$, we deduce $x_{1} v_{-2}=0$, hence $x_{3} v_{-2}=\left[x_{1} x_{2}\right] v_{-2}=0$ and $x_{i} v_{-2}=0 \forall i \geqq 3$. Thus $\left\{v_{-2}\right\}$ would be the trivial module over the subalgebra $\mathscr{V}_{3}$ and this is impossible. We deduce $x_{-2} v_{0}=0$, then $\left\{v_{0}\right\}$ is the trivial $\mathscr{V}$-module.

If $x_{1} v_{1}=0$, then $v_{0}=x_{-1} v_{1} \neq 0$ and $x_{-1} v_{0} \neq 0$. As $Q_{1} v_{1}=2 v_{1}$, we get $Q_{1} v_{0}=2 v_{0}$ hence $x_{1} v_{0} \neq 0$. Consequently there exists $v_{0}^{\prime} \in \mathscr{A}_{0} \cap \operatorname{Ker} x_{1} \cap \operatorname{Ker} x_{-1}$. From the 
relation $\left[x_{-2} x_{1}\right] v_{0}^{\prime}=0$, by the same arguments as in the previous case we get $x_{-2} v_{0}^{\prime}=0$. Thus, $\left\{v_{0}^{\prime}\right\}$ is the trivial $\mathscr{V}$-submodule.

If $x_{-1} v_{-1}=0$, the proof is similar.

Theorem (III.8). Let $\mathscr{A}$ be an indecomposable bounded admissible $\mathscr{V}$-module with an asymptotic dimension $q$ and such that $\operatorname{Ker} x_{1}+\operatorname{Ker} x_{-1} \neq\{0\}$. Then there exists at most one weight space with a dimension different from $q$. Its weight is necessarily zero. Moreover if $\operatorname{dim} \mathscr{A}_{0}>q, q \in \mathbb{N}^{*}$, then there exists in $\mathscr{A}$ a proper $\mathscr{V}$-submodule with an asymptotic dimension 0 or 1 .

Proof. The first part of the theorem is given by Corollary (III.3) for $q=0$. Let $\mathscr{A}$ be a $\mathscr{V}$-module satisfying the assumptions of the theorem. Assume there exists, at least, a weight space with a dimension different from $q\left(q \in \mathbb{N}^{*}\right)$. Set:

$$
n_{1}=\operatorname{Sup}\left\{n / \operatorname{dim} \mathscr{A}_{a+n} \neq q\right\}, \quad n_{2}=\operatorname{Inf}\left\{n / \operatorname{dim} \mathscr{A}_{a+n} \neq q\right\} .
$$

First case: $n_{1}=n_{2}=n_{0}$.

We shall prove by induction on $q$ that $a+n_{0}=0$. The case $q=1$ results from Theorem (III.5). As Ker $x_{-1} \neq\{0\}$, we consider $m_{0}=\operatorname{Sup}\left\{n / \operatorname{Ker} x_{-1} \cap \mathscr{A}_{a+n} \neq\{0\}\right\}$ and $v \in \mathscr{A}_{a+m_{0}}$ such that $x_{-1} v=0$.

- If the assumptions of (III.1) are satisfied by $v$, then from (III.1) and (III.2) there exists a $\mathscr{V}$-submodule $\mathscr{A}^{\prime}$ with an asymptotic dimension one. If all the weight spaces of $\mathscr{A}^{\prime}$ are one-dimensional, then $\mathscr{A} / \mathscr{A}^{\prime}$ verifies the induction hypothesis for $(q-1)$ and we conclude $a+n_{0}=0$. Assume now $\mathscr{A}^{\prime}$ has a zero weight space with a dimension different from one (necessarily $a \in Z$ ). Suppose $a+n_{0} \neq 0$ and consider the submodule $\bigoplus_{k \in Z}\left(\mathscr{A} / \mathscr{A}^{\prime}\right)_{a+n_{0}+k\left(\left|a+n_{0}\right|+1\right)}$ over the subalgebra $\mathscr{V}_{\left|a+n_{0}\right|+1}$. By (III.5) it satisfies the induction hypothesis for $(q-1)$ and we get $a+n_{0}=0$ which is a contradiction. In this case, the existence of $\mathscr{A}^{\prime}$ and the theorem (III.5) prove the second part of the theorem for all $q \in \mathbb{N}^{*}$.

- If $v$ does not satisfy the assumptions of (III.1), then there exists $m_{1} \in \mathbb{N}$ such that $x_{1}^{m_{1}+1} v=0$ et $x_{1}^{m_{1}} v \notin x_{-1}\left(\mathscr{A}_{a+m_{0}+m_{1}+1}\right)$. As $x_{-1}$ is injective on $\mathscr{A}_{a+m_{0}+n+1} \forall n \in \mathbb{N}$, we deduce $\operatorname{dim} \mathscr{A}_{a+m_{0}+n+1}<\operatorname{dim} \mathscr{A}_{a+m_{0}+m_{1}} \forall n \geqq m_{1}$. Thus, $\mathscr{A}_{a+m_{0}+m_{1}}$ is the only weight space with a dimension strictly higher than $q$. Hence $m_{0}+m_{1}=n_{0}$. As $n_{0} \leqq m_{0}$, we deduce $n_{0}=m_{0}$ and $m_{1}=0$. Thus we have $x_{1} v=0$ and $a+n_{0}=0$. The relation $\left[x_{-1} x_{2}\right] v=3 x_{1} v=0$ gives $x_{2} v=0 .\{v\}$ is the trivial $\mathscr{A}$-module and this proves in this case the second part of the theorem for $q \in \mathbb{N}^{*}$.

Second case: $n_{2}<n_{1}$.

By the same arguments as in the proof of Theorem (III.5) this case is impossible.

Theorem (III.9). Let $\mathscr{A}$ be an irreducible bounded admissible $\mathscr{V}$-module where $\operatorname{Ker} x_{1}+\operatorname{Ker} x_{-1} \neq\{0\}$. Then all its weight spaces have a dimension less than or equal to one.

Proof. Let $\mathscr{A}$ be an indecomposable bounded admissible $\mathscr{V}$-module where $\operatorname{Ker} x_{1}+\operatorname{Ker} x_{-1} \neq\{0\}$. Let $q$ be its asymptotic dimension. Suppose $q \geqq 2$. If there exists in $\mathscr{A}$ a zero weight space with a dimension strictly higher than $q$, then by (III.8), $\mathscr{A}$ is not irreducible. Otherwise, as we can consider the inverse module, we may suppose $\operatorname{Ker} x_{-1} \neq\{0\}$. Then there exists $v$ satisfying the assumptions of (III.1). From (III.1) and (III.2) we deduce that $\mathscr{A}$ is not irreducible. Thus, if $\mathscr{A}$ is irreducible, we have $q \leqq 1$. Then the result is given by (III.3) for $q=0$ and by (III.5) for $q=1$. 


\section{Bounded Admissible $\mathscr{V}$-Modules Where $\operatorname{Ker} \boldsymbol{x}_{-1}+\operatorname{Ker} \boldsymbol{x}_{1}=\{0\}$}

In this case, writing the asymptotic relation (II.8) for $k=0$, we relate $A(0)$ to $B(2)$. From (II.3), we deduce a relation between $B(2)$ and $B(0)$. Thus we get:

$$
\begin{aligned}
& \Gamma(a+3-\Lambda) \Gamma(a-2+\Lambda) A(0)+\prod_{-2}^{+\infty}\left(1+\frac{\Delta(i)}{a+i+\Lambda}\right) B(0) \prod_{0}^{+\infty}\left(1+\frac{\Delta(i)}{a+i+\Lambda}\right)^{-1} \\
& \cdot \Gamma(a+\Lambda) \Gamma(a+1-\Lambda)=\Gamma(a+2-\Lambda) \Gamma(a-2+\Lambda) \\
& \cdot\left\{\left[4 a+2+2 \Lambda(\Lambda-1)\left(\frac{1}{a-\Lambda}+\frac{1}{a-1-\Lambda}\right)\right] \Delta(0)\right. \\
& \left.\quad-\left[\frac{\Lambda}{a-\Lambda}-\frac{\Lambda-1}{a-1-\Lambda}\right](a+1-\Lambda) \Delta^{2}(0)\right\}
\end{aligned}
$$

This equation has been obtained from the commutation relation $\left[x_{-2} x_{2}\right]=4 x_{0}+\frac{1}{2} c$ applied to $\mathscr{A}_{a+n}$ by letting $n \rightarrow \infty$. Here we can also let $n \rightarrow-\infty$. This leads to:

$$
\begin{aligned}
A(0) & \Gamma(-a+1-\Lambda) \Gamma(-a+\Lambda)+\Gamma(-a+3-\Lambda) \Gamma(-a-2+\Lambda) \\
& \cdot \prod_{-3}^{+\infty}\left(1+\frac{\Delta(i)}{a+i+\Lambda}\right)^{-1} B(0) \prod_{-1}^{-\infty}\left(1+\frac{\Delta(i)}{a+i+\Lambda}\right) . \\
= & -\frac{\Gamma(-a+1-\Lambda) \Gamma(-a+\Lambda)}{(-a+\Lambda-2)}\left\{\left[4 a+2+2 \Lambda(\Lambda-1)\left(\frac{1}{a-\Lambda}+\frac{1}{a-1-\Lambda}\right)\right] \Delta(0)\right. \\
& \left.-\left[\frac{\Lambda}{a-\Lambda}-\frac{\Lambda-1}{a-1-\Lambda}\right](a+1-\Lambda) \Delta^{2}(0)\right\} .
\end{aligned}
$$

Using the relation $\Gamma(\xi) \Gamma(1-\xi)=\frac{\pi}{\sin (\pi \xi)}$, from Eqs. (IV.1) and (IV.2) it follows:

$$
\begin{aligned}
A(0) & \prod_{-\infty}^{+\infty}\left(1+\frac{\Delta(i)}{a+i+\Lambda}\right) \sin (\pi(a+\Lambda)) \sin (\pi(-a+\Lambda)) \\
& -\sin (\pi(a+\Lambda)) \sin \left(\pi(-a+\Lambda) \prod_{-\infty}^{+\infty}\left(1+\frac{\Delta(i)}{a+i+\Lambda}\right) A(0)=0 .\right.
\end{aligned}
$$

The functions $\sin (\pi(a+\Lambda))$ and $\sin (\pi(-a+\Lambda))$ here denote the obvious diagonal matrices.

1. Elementary Properties About $\pi=\prod_{-\infty}^{+\infty}\left(1+\frac{\Delta(i)}{a+i+\Lambda}\right)$. Let $\mathscr{A}=\bigoplus_{i=1}^{p} N_{i}$ be the decomposition of $\mathscr{A}$, where $N_{l}$ is the characteristic subspace of $Q_{1}$ corresponding to the eigenvalue $\Lambda_{l}\left(\Lambda_{l}-1\right)$. Let us recall that $\left(a+1-\Lambda_{l}\right) \Delta_{l}(0)$ is the matrix of the restriction to $N_{l}$ of the nilpotent part $(a+1-\Lambda) \Delta(0)$ of $-Q_{1}$ on the chosen Jordan basis in $\mathscr{A}_{0}$ (I.2.5). The submatrix $\pi_{l}$ of $\pi$ restricted to $N_{l}$ can be developed as follows:

$$
\pi_{l}=\mathrm{Id}+\sum_{j=2}^{+\infty} \alpha_{j}(l)\left(\left(a+1-\Lambda_{l}\right) \Delta_{l}(0)\right)^{j-1}
$$


where

$$
\begin{aligned}
& \alpha_{2}(l)=\sum_{-\infty}^{+\infty} \frac{1}{\left(a+i+1-\Lambda_{l}\right)\left(a+i+\Lambda_{l}\right)}, \\
& \alpha_{3}(l)=\sum_{\substack{i=-\infty \\
i<j}}^{+\infty} \sum_{j=-\infty}^{+\infty}\left(\frac{1}{\left(a+i+1-\Lambda_{l}\right)\left(a+i+\Lambda_{l}\right)}\right)\left(\frac{1}{\left(a+j+1-\Lambda_{l}\right)\left(a+j+\Lambda_{l}\right)}\right) .
\end{aligned}
$$

(a) Case $\Lambda_{l} \neq \frac{1}{2}$ :

$$
\alpha_{2}(l)=\frac{\pi}{2 \Lambda_{l}-1}\left[\operatorname{cotg}\left(\dot{\pi}\left(a-\Lambda_{l}\right)\right)-\operatorname{cotg}\left(\pi\left(a+\Lambda_{l}\right)\right)\right] .
$$

Thus we have:

$$
\alpha_{2}(l) \neq 0 \Leftrightarrow 2 \Lambda_{l} \notin Z
$$

For $2 \Lambda_{l} \in Z$, we get:

$$
\begin{aligned}
2 \alpha_{3}(l)= & \left(\sum_{-\infty}^{+\infty} \frac{1}{\left(a+i+1-\Lambda_{l}\right)\left(a+i+\Lambda_{l}\right)}\right)^{2}-\sum_{-\infty}^{+\infty} \frac{1}{\left(a+i+1-\Lambda_{l}\right)^{2}\left(a+i+\Lambda_{l}\right)^{2}} \\
= & \alpha_{2}(l)^{2}-\frac{1}{\left(1-2 \Lambda_{l}\right)^{2}} \sum_{-\infty}^{+\infty}\left(\frac{1}{\left(a+i+1-\Lambda_{l}\right)^{2}}+\frac{1}{\left(a+i+\Lambda_{l}\right)^{2}}\right) \\
& +\beta\left[\sum_{-\infty}^{+\infty} \frac{1}{a+i-\Lambda_{l}}-\sum_{-\infty}^{+\infty} \frac{1}{a+i+\Lambda_{l}}\right],
\end{aligned}
$$

where

$$
\sum_{-\infty}^{+\infty} \frac{1}{a+i-\Lambda_{l}}-\sum_{-\infty}^{+\infty} \frac{1}{a+i+\Lambda_{l}}=0
$$

Thus:

$$
\begin{array}{ll}
\alpha_{3}(l)=-\frac{1}{\left(1-2 \Lambda_{l}\right)^{2}} \sum_{-\infty}^{+\infty}\left(\frac{1}{a+i}\right)^{2}=\frac{-1}{\left(1-2 \Lambda_{l}\right)^{2}} \frac{\pi^{2}}{\sin ^{2}(\pi a)} \quad \text { for } \quad \Lambda_{l} \in Z, \\
\alpha_{3}(l)=-\frac{1}{\left(1-2 \Lambda_{l}\right)^{2}} \sum_{-\infty}^{+\infty}\left(\frac{1}{a+i+\frac{1}{2}}\right)^{2}=\frac{-1}{\left(1-2 \Lambda_{l}\right)^{2}} \frac{\pi^{2}}{\sin ^{2}\left(\pi\left(a+\frac{1}{2}\right)\right)} & \text { for } \quad \Lambda_{l} \notin Z .
\end{array}
$$

In both cases $\alpha_{3}(l) \neq 0$.

(b) Case $\Lambda_{l}=\frac{1}{2}$. Then

$$
\alpha_{2}(l)=\sum_{-\infty}^{+\infty}\left(\frac{1}{a+i+\frac{1}{2}}\right)^{2}=\frac{\pi^{2}}{\sin ^{2}\left(\pi\left(a+\frac{1}{2}\right)\right)} \neq 0 .
$$

2. Necessary Condition for Indecomposability of a Bounded Admissible $\mathscr{V}$-Module $\mathscr{A}$ where $\operatorname{Ker} x_{-1}+\operatorname{Ker} x_{1}=\{0\}$. Let $A_{i j}(n)$ (respectively $\left.B_{i j}(n)\right), i \leqq i \leqq p, 1 \leqq j \leqq p$, be the blocks of the matrices $A_{i j}(n)$ (respectively $\left.B_{i j}(n)\right)$ relative to the decomposition $\mathscr{A}=\bigoplus_{i=1}^{P} N_{i}$. By considering the $(i, j)$ entries of Eq. (IV.3) we get: 


$$
\begin{aligned}
A_{i j}(0) & \prod_{-\infty}^{+\infty}\left(1+\frac{\Delta_{j}(k)}{a+k+\Lambda_{j}}\right) \sin \left(\pi\left(a+\Lambda_{j}\right)\right) \sin \left(\pi\left(-a+\Lambda_{j}\right)\right) \\
- & \sin \left(\pi\left(a+\Lambda_{i}\right)\right) \sin \left(\pi\left(-a+\Lambda_{i}\right)\right) \\
& \prod_{-\infty}^{+\infty}\left(1+\frac{\Delta_{i}(k)}{a+k+\Lambda_{i}}\right) A_{i j}(0)=0 .
\end{aligned}
$$

Assume the first column of $A_{i j}(0)$ is non-zero. Let $b$ be the non-zero element of this column with highest line index. Looking at the corresponding element in the matrix equality (IV.4) we get:

$$
b\left[\sin \left(\pi\left(a+\Lambda_{j}\right)\right) \sin \left(\pi\left(-a+\Lambda_{j}\right)\right)-\sin \left(\pi\left(a+\Lambda_{i}\right)\right) \sin \left(\pi\left(-a+\Lambda_{i}\right)\right)\right]=0 .
$$

We have:

$$
\cos \left(2 \pi \Lambda_{i}\right)=\cos \left(2 \pi \Lambda_{j}\right) \Leftrightarrow \Lambda_{i} \pm \Lambda_{j} \in Z .
$$

Thus, for $\Lambda_{i} \pm \Lambda_{j} \notin Z$ we deduce that the first column of $A_{i j}(0)$ is zero. Iterating the proof on the next columns, we get $A_{i j}(0)=0$ for all $i$ and $j$ such that $\Lambda_{i} \pm \Lambda_{j} \notin Z$.

From Eq. (IV.1) it follows $B_{i j}(0)=0$ for all $i$ and $j$ such that $\Lambda_{i} \pm \Lambda_{j} \notin Z$. And finally from (II.2) and (II.3), we get:

$$
A_{i j}(n)=B_{i j}(n)=0 \quad \forall n \in Z \quad \text { for all } i \text { and } j \text { such that } \quad \Lambda_{i} \pm \Lambda_{j} \notin Z .
$$

From this, it results that in a bounded admissible indecomposable $\mathscr{V}$-module the eigenvalues $\Lambda_{i}\left(\Lambda_{i}-1\right)$ of $Q_{1}$ necessarily satisfy $\Lambda_{i} \pm \Lambda_{j} \in Z$ for all $i$ and $j$. Furthermore, we know that we can change $\Lambda_{i}$ to $\left(1-\Lambda_{i}\right)$ so that we get the following:

Proposition (IV.5). Let us consider an indecomposable bounded admissible $\mathscr{V}$-module such that $\operatorname{Ker} x_{-1}+\operatorname{Ker} x_{1}=\{0\}$. Then, the eigenvalues $\Lambda_{i}\left(\Lambda_{i}-1\right)$ of $Q_{1}$ can be chosen so that $\Lambda_{j}-\Lambda_{i} \in Z$ for all $i$ and $j$.

3. Necessary Condition for Irreducibility of a Bounded Admissible $\mathscr{V}$-Module $\mathscr{A}$ where $\operatorname{Ker} x_{-1}+\operatorname{Ker} x_{1}=\{0\}$. In view of Proposition (IV.5), we can suppose that the eigenvalues $\Lambda_{i}\left(\Lambda_{i}-1\right), 1 \leqq i \leqq p$, of $Q_{1}$ satisfy $\Lambda_{i}-\Lambda_{j} \in Z \forall i \neq j$. We shall now prove the following:

Theorem (IV.6). Let $\mathscr{A}$ be an indecomposable bounded admissible $\mathscr{V}$-module such that $\operatorname{Ker} x_{-1}+\operatorname{Ker} x_{1}=\{0\}$. Then:

a) if $2 \Lambda_{i} \notin Z, \bigoplus_{i=1}^{p} \operatorname{Ker}\left[Q_{1}-\Lambda_{i}\left(\Lambda_{i}-1\right)\right]$ is a $\mathscr{V}$-submodule of $\mathscr{A}$.

b) if $2 \Lambda_{i} \in Z, \bigoplus_{i=1}^{p} \operatorname{Ker}\left[Q_{1}-\Lambda_{i}\left(\Lambda_{i}-1\right)\right]^{q_{i}}$, where $q_{i}=2$ if $2 \Lambda_{i} \neq 1$ and $q_{i}=1$ if $2 \Lambda_{i}=1$ is a $\mathscr{V}$-submodule of $\mathscr{A}$.

Equation (IV.3) here gives:

$$
A(0) \prod_{-\infty}^{+\infty}\left(1+\frac{\Delta(i)}{a+i+\Lambda}\right)-\prod_{-\infty}^{+\infty}\left(1+\frac{\Delta(i)}{a+i+\Lambda}\right) A(0)=0
$$

From this, it results that the eigenspace of

$$
\pi=\prod_{-\infty}^{+\infty}\left(1+\frac{\Delta(i)}{a+i+\Lambda}\right)
$$


is invariant by $A(0)$. As the characteristic subspaces $N_{i}$ are invariant by $\pi$, we only need to determine the eigenspaces of the restrictions $\pi_{l}=\pi_{\mid N_{l}}$. In case where $\alpha_{2}(l) \neq 0$, it is clear that the eigenvectors of $\pi_{l}$ are those of $\Delta_{l}(0)$, that is to say those of $Q_{1}$. In case where $\alpha_{2}(l)=0$, we have always $\alpha_{3}(l) \neq 0$ so that the eigenvectors of $\pi_{l}$ are those of $\Delta_{l}^{2}(0)$ which are also those of $\left[Q_{1}-\Lambda_{l}\left(\Lambda_{l}-1\right)\right]^{2}$. From our results on $\alpha_{2}(l)$ and $\alpha_{3}(l)$, we get invariant subspaces by $A(0)$ :

$$
\bigoplus_{i=1}^{p} \operatorname{Ker}\left[Q_{1}-\Lambda_{i}\left(\Lambda_{i}-1\right)\right] \text { when } 2 \Lambda_{i} \notin Z \forall i
$$

$$
\operatorname{Ker}\left[Q_{1}-\Lambda_{i}\left(\Lambda_{i}-1\right)\right]^{q_{i}} \quad \text { with } \quad q_{i}=2 \text { if } 2 \Lambda_{i} \neq 1 \text { and } q_{i}=1 \text { if } 2 \Lambda_{i}=1
$$

when $2 \Lambda_{i} \in Z \forall i$.

From Eq. (IV.1) relating $A(0)$ and $B(0)$, these subspaces are invariant by $B(0)$. Then from (II.2) and (II.3) follows their invariance by $A(n)$ and $B(n)$ for all $n \in Z$. Thus these subspaces are invariant by $x_{-2}$ and $x_{2}$ and hence by $\mathscr{V}$.

4. Proof of the Conjecture of $V$-Kac for Bounded Admissible $\mathscr{V}$-Module where $\operatorname{Ker} x_{-1}+\operatorname{Ker} x_{1}=\{0\}$. We need here to return to Eq. (II.5). This equation comes from the commutation relation $\left[x_{-2} x_{2}\right]=4 x_{0}+\frac{1}{2} c$ applied to $\mathscr{A}_{a+n}$. The relation (II.2) gives:

$$
A(n)=\frac{\Gamma(a+3-\Lambda)}{\Gamma(a+n+3-\Lambda)} A(0) \frac{\Gamma(a+n+1-\Lambda)}{\Gamma(a+1-\Lambda)}+E(n)
$$

where

$$
E(n)=\frac{3 n(a+1-\Lambda) \Delta(0)}{(a+n+2-\Lambda)(a+n+1-\Lambda)} .
$$

The relations (II.3) and (IV.1) give:

where

$$
\begin{aligned}
B(n)= & -\Gamma(a+3-\Lambda) \Gamma(a+n-2+\Lambda) \prod_{n-2}^{\infty}\left(1+\frac{\Delta(i)}{a+i+\Lambda}\right)^{-1} A(0) \\
& \cdot \prod_{n}^{\infty}\left(1+\frac{\Delta(i)}{a+i+\Lambda}\right) \frac{1}{\Gamma(a+1-\Lambda) \Gamma(a+n+\Lambda)}+H^{\prime}(n),
\end{aligned}
$$

$$
\begin{aligned}
H^{\prime}(n)= & (a+n-2+\Lambda+\Delta(n-2))^{-1}(a+n-1+\Lambda+\Delta(n-1))^{-1} \\
& \cdot\left\{\left[4 a+2+n+\frac{2 \Lambda(\Lambda-1)}{a+n-\Lambda}+\frac{2 \Lambda(\Lambda-1)}{a+n-1-\Lambda}\right](a+1-\Lambda) \Delta(0)\right. \\
& \left.+\left[\frac{-\Lambda}{a+n-\Lambda}+\frac{\Lambda-1}{a+n-1-\Lambda}\right](a+1-\Lambda)^{2} \Delta^{2}(0)\right\} .
\end{aligned}
$$

Substituting (IV.7) and (IV.8) in (II.5), we get:

$$
\begin{aligned}
A(0) & \frac{1}{(a+n-\Lambda)(a+n-1-\Lambda)}\left(a+n-2 \Lambda+H^{\prime}(n)\right) \\
& -\left(a+n+2-2 \Lambda+H^{\prime}(n+2)\right) \frac{1}{(a+n+2-\Lambda)(a+n+1-\Lambda)} A(0)
\end{aligned}
$$




$$
\begin{aligned}
& +\Gamma(a+n+1-\Lambda) \Gamma(a+n+\Lambda) \prod_{n}^{\infty}\left(1+\frac{\Delta(i)}{a+i+\Lambda}\right)^{-1} A(0) \prod_{n+2}^{\infty}\left(1+\frac{\Delta(i)}{a+i+\Lambda}\right) \\
& \cdot \frac{1}{\Gamma(a+n+1-\Lambda) \Gamma(a+n+2+\Lambda)}(a+n+2 \Lambda+E(n)) \\
& -(a+n-2+2 \Lambda+E(n-2)) \Gamma(a+n+1-\Lambda) \Gamma(a+n-2+\Lambda) \\
& \cdot \prod_{n-2}^{\infty}\left(1+\frac{\Delta(i)}{a+i+\Lambda}\right)^{-1} A(0) \prod_{n}^{\infty}\left(1+\frac{\Delta(i)}{a+i+\Lambda}\right) \frac{1}{\Gamma(a+n+1-\Lambda) \Gamma(a+n+\Lambda)} \\
& =-\Gamma(a+n+1-\Lambda) \Gamma(a+n+\Lambda) \prod_{n}^{\infty}\left(1+\frac{\Delta(i)}{a+i+\Lambda}\right)^{-1} A(0) \\
& +\prod_{n+2}^{\infty}\left(1+\frac{\Delta(i)}{a+i+\Lambda}\right) \frac{(a+2-\Lambda)(a+1-\Lambda)}{\Gamma(a+n+2+\Lambda) \Gamma(a+n+3-\Lambda)} A(0) \\
& +A(0) \Gamma(a+n-1-\Lambda) \Gamma(a+n-2+\Lambda)(a+2-\Lambda)(a+1-\Lambda) \\
& \cdot \prod_{n-2}^{\infty}\left(1+\frac{\Delta(i)}{a+i+\Lambda}\right)^{-1} A(0) \prod_{n}^{\infty}\left(1+\frac{\Delta(i)}{a+i+\Lambda}\right) \frac{1}{\Gamma(a+n+1-\Lambda) \Gamma(a+n+\Lambda)} \\
& +\left(a+n+2-2 \Lambda+H^{\prime}(n+2)\right)(a+n+2 \Lambda+E(n)) \\
& -(a+n-2+2 \Lambda+E(n-2))\left(a+n-2 \Lambda+H^{\prime}(n)\right)-4(a+n) .
\end{aligned}
$$

\section{$A$-Case where $Q_{1}$ is Diagonalizable}

We always consider the decomposition $\mathscr{A}=\bigoplus_{i=1}^{p} N_{i}$. The eigenvalues $\Lambda_{i}\left(\Lambda_{i}-1\right)$ of $Q_{1}$ are chosen such that $\Lambda_{i}-\Lambda_{j} \in Z$ for all $i$ and $j$. Assume the indexes such that $\operatorname{Re} \Lambda_{1}<\operatorname{Re} \Lambda_{2}<\cdots<\operatorname{Re} \Lambda_{p}$. Moreover in the case where $2 \Lambda_{i} \in Z$, as we can change $\Lambda_{i}$ to $\left(1-\Lambda_{i}\right)$, we can suppose that $\operatorname{Re} \Lambda_{i} \leqq \frac{1}{2} \forall i$.

Since $Q_{1}$ is diagonalizable $\Delta(n)=E(n)=H^{\prime}(n)=0 \forall n$. By considering the $(k, j)$ entries of Eq. (IV.9), we get:

$$
\begin{aligned}
A_{k j}(0) & \left(\frac{a+n-2 \Lambda_{j}}{\left(a+n-\Lambda_{j}\right)\left(a+n-1-\Lambda_{j}\right)}-\frac{a+n+2-2 \Lambda_{k}}{\left(a+n+2-\Lambda_{k}\right)\left(a+n+1-\Lambda_{k}\right)}\right) \\
& +\frac{\Gamma\left(a+n+1-\Lambda_{k}\right) \Gamma\left(a+n+\Lambda_{k}\right)}{\Gamma\left(a+n+1-\Lambda_{j}\right) \Gamma\left(a+n+\Lambda_{j}\right)} \\
& \cdot\left(\frac{a+n+2 \Lambda_{j}}{\left(a+n+1+\Lambda_{j}\right)\left(a+n+\Lambda_{j}\right)}-\frac{a+n-2+2 \Lambda_{k}}{\left(a+n-1+\Lambda_{k}\right)\left(a+n-2+\Lambda_{k}\right)}\right) A_{k j}(0) \\
= & -\Gamma\left(a+n+1-\Lambda_{k}\right) \Gamma\left(a+n+\Lambda_{k}\right) \sum_{i=1}^{p} \frac{\left(a+2-\Lambda_{i}\right)\left(a+1-\Lambda_{i}\right)}{\Gamma\left(a+n+2+\Lambda_{i}\right) \Gamma\left(a+n+3-\Lambda_{i}\right)} \\
& \cdot A_{k i}(0) A_{i j}(0)+\frac{1}{\Gamma\left(a+n+1-\Lambda_{j}\right) \Gamma\left(a+n+\Lambda_{j}\right)} \\
& \cdot \sum_{i=1}^{p}\left(a+2-\Lambda_{i}\right)\left(a+1-\Lambda_{i}\right) \Gamma\left(a+n-1-\Lambda_{i}\right) \Gamma\left(a+n-2+\Lambda_{i}\right) A_{k i}(0) A_{i j}(0) .
\end{aligned}
$$


As $\left(\Lambda_{i}-\Lambda_{j}\right) \in Z$ for all $i$ and $j$, the matrices of this equation are rational functions. By considering the $(k, 1)$ entries and the residue at the pole $\left(-a-2+\Lambda_{1}\right)$ we get:

$$
A_{k 1}(0) A_{11}(0)=0 \quad \forall k>1 .
$$

By considering the same pole $\left(-a-2+\Lambda_{1}\right)$ relative to the $(1, j)$ entries for $j \geqq 1$, we are lead to the following discussion:

(a) $\Lambda_{1} \neq 0$ and $2 \Lambda_{1} \neq 1$

$\left(-a-2+\Lambda_{1}\right)$ is a pole of order 1 in Eq. (IV.10) for all $k$ and $j$. Thus we get:

$$
A_{11}(0) A_{1 j}(0)=\frac{2 \Lambda_{1}\left(2 \Lambda_{1}-1\right)\left(1-\Lambda_{1}\right)}{\left(a+2-\Lambda_{1}\right)\left(a+1-\Lambda_{1}\right)} A_{1 j}(0) \quad \forall j \geqq 1 .
$$

In particular, from the relation

$$
A_{11}^{2}(0)=\frac{2 \Lambda_{1}\left(2 \Lambda_{1}-1\right)\left(1-\Lambda_{1}\right)}{\left(a+2-\Lambda_{1}\right)\left(a+1-\Lambda_{1}\right)} A_{11}(0)
$$

we deduce that the matrix $A_{11}(0)$ is diagonalizable with only two possible eigenvalues 0 and $a\left(\Lambda_{1}\right)=\frac{2 \Lambda_{1}\left(2 \Lambda_{1}-1\right)\left(1-\Lambda_{1}\right)}{\left(a+2-\Lambda_{1}\right)\left(a+1-\Lambda_{1}\right)}$. Let $n_{1}$ be the rank of the matrix
$A_{11}(0)$ :

a) If $n_{1}=0$, we obtain $A_{1 j}=0 \forall j$.

- For $p>1$ : the relation (IV.1) gives $B_{1 j}(0)=0 \forall j$. From relations (IV.7) and (IV.8) $B_{1 j}(n)=A_{1 j}(n)=0 \forall j$ and $\forall n \in Z$ results. From this, we deduce that the subspace $\bigoplus_{i=2}^{p} N_{i}$ is invariant by $x_{-2}$ and $x_{2}$, hence by the algebra $\mathscr{V}$.

- For $p=1: A(0)=0$ and all vectors of $\mathscr{A}_{a} \cap N_{1}$ satisfy the conditions of Theorem (II.10). Thus, there exists a $\mathscr{V}$-submodule with an asymptotic dimension 1. Hence all its weight spaces are one dimensional.

$\beta$ ) If $n_{1} \neq 0$, from relations $A_{k 1}(0) A_{11}(0)=0 \forall k>1$, we deduce that the image of $A_{11}(0)$ is included in the kernel of $A_{k 1}(0)$ for all $k>1$. From this, the existence in $N_{1} \cap \mathscr{A}_{a}$ of a common eigenvector $v$ for both $A_{11}(0)$ and $A(0)$ results. Thus $x_{2} v$ is a multiple of $x_{1}^{2} v$ and Theorem (II.10) asserts the existence of a $\mathscr{V}$-submodule with an asymptotic dimension 1 . Hence, all its weight-spaces are one-dimensional.

(b) $\Lambda_{1}=0$ or $2 \Lambda_{1}=1$

$\Lambda_{1}\left(\Lambda_{1}-1\right)$ is here the only eigenvalue of $Q_{1}$ and $\mathscr{A}=N_{1}$.

For $\Lambda_{1}=0$, the pole $\left(-a-2+\Lambda_{1}\right)$ only appears in the second member of Eq. (IV.10) and we get $A_{11}^{2}(0)=0$. For $2 \Lambda_{1}=1,\left(-a-2+\Lambda_{1}\right)$ is a pole of order 2 in the second member of (IV.10) and we also get $A_{11}^{2}(0)=0$. In both cases there exists an eigenvector of $A(0)=A_{11}(0)$ and we apply Theorem (II.10).

\section{$B$-Case where $Q_{1}$ is not Diagonalizable}

Assume here $\mathscr{A}$ is an irreducible bounded admissible $\mathscr{V}$-module such that $\operatorname{Ker} x_{1}+\operatorname{Ker} x_{-1}=\{0\}$ and at least one characteristic subspace of $Q_{1}$ is of order 2. From Theorem (IV.6), it results that $2 \Lambda_{i} \in Z \forall i$ and all the characteristic subspaces of $Q_{1}$ are of order less than or equal to 2 so that $\Delta^{2}(0)=0$. We suppose always 
$\Lambda_{i}<\Lambda_{i+1} \leqq \frac{1}{2} \forall i$. By considering the decomposition $\mathscr{A}=\bigoplus_{i=1}^{p} N_{i}$, the $(k, l)$ entries of Eq. (IV.9) give:

$$
\begin{aligned}
A_{k l}(0) & \frac{1}{\left(a+n-\Lambda_{l}\right)\left(a+n-1-\Lambda_{l}\right)}\left(a+n-2 \Lambda_{l}+H^{\prime}(n)\right)-\left(a+n+2-2 \Lambda_{l}+H_{k k}^{\prime}(n+2)\right) \\
& \cdot \frac{1}{\left(a+n+2-\Lambda_{k}\right)\left(a+n+1-\Lambda_{k}\right)} A_{k l}(0)+\frac{\Gamma\left(a+n+1-\Lambda_{k}\right) \Gamma\left(a+n+\Lambda_{k}\right)}{\Gamma\left(a+n+1-\Lambda_{l}\right) \Gamma\left(a+n+2+\Lambda_{l}\right)} \\
& \cdot \prod_{n}^{\infty}\left(1+\frac{\Delta_{k}(i)}{a+i+\Lambda_{k}}\right)^{-1} A_{k l}(0) \prod_{n+2}^{\infty}\left(1+\frac{\Delta_{l}(i)}{a+i+\Lambda_{l}}\right)\left(a+n+2 \Lambda_{l}+E_{l l}(n)\right) \\
& -\left(a+n-2+2 \Lambda_{k}+E_{k k}(n-2)\right) \frac{\Gamma\left(a+n+1-\Lambda_{k}\right) \Gamma\left(a+n-2+\Lambda_{k}\right)}{\Gamma\left(a+n+1-\Lambda_{l}\right) \Gamma\left(a+n+\Lambda_{l}\right)} \\
& \cdot \prod_{n-2}^{\infty}\left(1+\frac{\Delta_{k}(i)}{a+i+\Lambda_{k}}\right)^{-1} A_{k l}(0) \prod_{n}^{\infty}\left(1+\frac{\Delta_{l}(i)}{a+i+\Lambda_{l}}\right) \\
= & -\Gamma\left(a+n+1-\Lambda_{k}\right) \Gamma\left(a+n+\Lambda_{k}\right) \prod_{n}^{\infty}\left(1+\frac{\Delta_{k}(i)}{a+i+\Lambda_{k}}\right) \sum_{j=1}^{p} A_{k j}(0) \\
& \cdot \prod_{n+2}^{\infty}\left(1+\frac{\Delta_{j}(i)}{a+i+\Lambda_{j}}\right) A_{j l}(0) \frac{\left(a+2-\Lambda_{j}\right)\left(a+1-\Lambda_{j}\right)}{\Gamma\left(a+n+2+\Lambda_{j}\right) \Gamma\left(a+n+3-\Lambda_{j}\right)} \\
& +\sum_{j=1}^{p} \Gamma\left(a+n-1-\Lambda_{j}\right) \Gamma\left(a+n-2+\Lambda_{j}\right)\left(a+2-\Lambda_{j}\right)\left(a+1-\Lambda_{j}\right) A_{k j}(0) \\
& \cdot \prod_{n-2}^{\infty}\left(1+\frac{\Delta_{j}(i)}{a+i+\Lambda_{j}}\right)^{-1} A_{j l}(0) \prod_{n}^{\infty}\left(1+\frac{\Delta_{l}(i)}{a+i+\Lambda_{l}}\right) \frac{1}{\Gamma\left(a+n+1-\Lambda_{l}\right) \Gamma\left(a+n+\Lambda_{l}\right)} \\
& +\left(a+n+2-2 \Lambda_{k}\right) E_{k l}(n)+H_{k l}^{\prime}(n+2)\left(a+n+2 \Lambda_{l}\right)-\left(a+n-2+2 \Lambda_{k}\right) H_{k l}^{\prime}(n) \\
& -E_{k l}(n-2)\left(a+n-2 \Lambda_{l}\right),
\end{aligned}
$$

where

$$
E_{k l}(n)=H^{\prime}{ }_{k l}(n)=0 \quad \forall k \neq l .
$$

The infinite products here are rational expressions of $n$. Indeed, for $2 \Lambda_{k} \neq 1$, we have:

$$
\begin{aligned}
& \prod_{n}^{\infty}\left(1+\frac{\left(a+1-\Lambda_{k}\right) \Delta_{k}(0)}{\left(a+i+1-\Lambda_{k}\right)\left(a+i+\Lambda_{k}\right)}\right) \\
& \quad=1+\left(a+1-\Lambda_{k}\right) \Delta_{k}(0) \sum_{i=n}^{\infty} \frac{1}{\left(a+i+1-\Lambda_{k}\right)\left(a+i+\Lambda_{k}\right)} \\
& \quad=1+\left(a+1-\Lambda_{k}\right) \Delta_{k}(0) \frac{1}{2 \Lambda_{k}-1} \sum_{n}^{\infty}\left[\frac{1}{a+i+1-\Lambda_{k}}-\frac{1}{a+i+\Lambda_{k}}\right] \\
& \quad=1-\frac{a+1-\Lambda_{k}}{2 \Lambda_{k}-1} \Delta_{k}(0) \sum_{i=n}^{n-2} \frac{1}{a+i+\Lambda_{k}} .
\end{aligned}
$$

For $2 \Lambda_{k}=1, \Delta_{k}(0)=0$ and the infinite products are the identity. All terms of (IV.11) are rational expressions of $n$. Let $j_{0}$ be the smallest index such that the corresponding characteristic subspace is of order 2 . 
In view of (IV.6) this imposes $2 \Lambda_{j_{0}} \neq 1$. Then for all $k,\left(-a-2+\Lambda_{j_{0}}\right)$ is a pole of order 2 for the unique following term in the second member of equality (IV.11):

$$
\begin{aligned}
- & \Gamma\left(a+n+1-\Lambda_{k}\right) \Gamma\left(a+n+\Lambda_{k}\right)\left(1+\frac{a+1-\Lambda_{k}}{2 \Lambda_{k}-1} \sum_{n}^{n-2 \Lambda_{k}} \frac{1}{a+i+\Lambda_{k}} \Delta_{k}(0)\right) A_{k j_{0}}(0) \\
& \cdot\left(1-\frac{a+1-\Lambda_{j_{0}}}{2 \Lambda_{j_{0}}-1} \sum_{n+2}^{n+2-2 \Lambda_{j_{0}}} \frac{1}{a+i+\Lambda_{j_{0}}} \Delta_{j_{0}}(0)\right) \\
& \cdot A_{j_{0} l}(0) \frac{\left(a+2-\Lambda_{j_{0}}\right)\left(a+1-\Lambda_{j_{0}}\right)}{\Gamma\left(a+n+2+\Lambda_{j_{0}}\right) \Gamma\left(a+n+3-\Lambda_{j_{0}}\right)} .
\end{aligned}
$$

And for $k=j_{0},\left(-a-2+\Lambda_{j_{0}}\right)$ is also a pole of order 2 in one term of the first member of (IV.11):

$$
-H_{j_{0} j_{0}}(n+2) \frac{1}{\left(a+n+2-\Lambda_{j_{0}}\right)\left(a+n+1-\Lambda_{j_{0}}\right)} A_{j_{0} l}(0) .
$$

By considering this pole of order 2 and multiplying on left by $\Delta_{k}(0)$, we get first:

$$
\Delta_{k}(0) A_{k j_{0}}(0) \Delta_{j_{0}}(0) A_{j_{0} l}(0)=0 \quad \forall k \quad \forall l .
$$

Then this gives:

$$
\begin{gathered}
A_{k j_{0}}(0) \Delta_{j_{0}}(0) A_{j_{0} l}(0)=0 \quad \forall k \neq j_{0} \\
A_{j_{0} j_{0}}(0) \Delta_{j_{0}}(0) A_{j_{0} l}(0)=\frac{2 \Lambda_{j_{0}}\left(2 \Lambda_{j_{0}}-1\right)\left(1-\Lambda_{j_{0}}\right)}{\left(a+2-\Lambda_{j_{0}}\right)\left(a+1-\Lambda_{j_{0}}\right)} \Delta_{j_{0}}(0) A_{j_{0} l}(0) .
\end{gathered}
$$

Assume, at first, there exists $l$ such that $\Delta_{j_{0}}(0) A_{j_{0} l}(0) \neq 0$. Then the image of $\Delta_{j_{0}}(0) A_{j_{0} l}(0)$ is included in the kernels of $A_{k j_{0}}$ for all $k \neq j_{0}$ and also in the eigenspace of $A_{j_{0} j_{0}}(0)$ relative to the eigenvalue $\frac{2 \Lambda_{j_{0}}\left(2 \Lambda_{j_{0}}-1\right)\left(1-\Lambda_{j_{0}}\right)}{\left(a+2-\Lambda_{j_{0}}\right)\left(a+1-\Lambda_{j_{0}}\right)}$. Thus an eigenvector $v$ of $A(0)$ exists in $N_{j_{0}} \cap\left[Q_{1}-\Lambda_{j_{0}}\left(\Lambda_{j_{0}}-1\right)\right]\left(N_{j_{0}}\right)$. Thus $x_{2} v$ is a multiple of $x_{1}^{2} v$ and Theorem (II.10) asserts the existence of a $\mathscr{V}$-submodule with an asymptotic dimension 1. Hence all its weight spaces are one dimensional. Assume now: $\Delta_{j_{0}}(0) A_{j_{0} l}(0)=0 \forall l$. It means that the images by $A(0)$ of all the vectors of $\mathscr{A}_{a}$ have components in $N_{j_{0}}$ which are eigenvectors of $Q_{1}$. Let us introduce the decomposition $N_{j_{0}}=N_{j_{0}}^{\prime} \oplus N^{\prime \prime}{ }_{j_{0}} \cdot N^{\prime} j_{0}$ is the eigenspace of $Q_{1}$ in $N_{j_{0}} \cdot N^{\prime \prime}{ }_{j_{0}}$ is the subspace of $N_{j_{0}}$ spanned by the characteristic vectors of $Q_{1}$ which are not eigenvectors in the given basis in $N_{j_{0}}$. In view of relations (IV.7) and (IV.8), from the condition $\Delta_{j_{0}}(0) A_{j_{0} l}(0)=0$ $\forall l$ we deduce $\Delta_{j_{0}}(n) A_{j_{0} l}(n)=0$ and $\Delta_{j_{0}}(n) B_{j_{0} l}(n)=0 \forall l$ and $\forall n$. From this it results that the subspace $N^{\prime}=N_{1} \oplus N_{2} \oplus \cdots \oplus N_{j_{0}-1} \oplus N^{\prime}{ }_{j_{0}} \oplus N_{j_{0}+1} \oplus \cdots \oplus N_{p}$ is invariant by $x_{-2}$ and $x_{2}$ and hence by $\mathscr{V}$. As $N^{\prime}{ }_{j 0} \neq\{0\}$ and $N^{\prime \prime}{ }_{j 0} \neq\{0\}, N^{\prime}$ is a non-zero proper $\mathscr{V}$-submodule.

In all cases we get a contradiction with the irreducibility hypothesis. Then:

Proposition (IV.12). Let us consider an irreducible bounded admissible $\mathscr{V}$-module such that $\operatorname{Ker} x_{-1}+\operatorname{Ker} x_{1}=\{0\}$. Then the Casimir $Q_{1}$ of $\mathscr{G}_{1}$ is diagonalizable.

From this proposition and the diagonalizable case, we get:

Theorem (IV.13). Let be an irreducible bounded admissible $\mathscr{V}$-module such that $\operatorname{Ker} x_{-1}+\operatorname{Ker} x_{1}=\{0\}$. Then the weight spaces are of dimension 1 . 
Theorems (IV.13) and (III.9) prove the conjecture of V. Kac for bounded admissible $\mathscr{V}$-modules: an irreducible bounded admissible $\mathscr{V}$-module has all its weight spaces of dimension less or equal to one.

Acknowledgements. We thank M. Flato for discussions and advice. We also thank L. J. Santharoubane for conversations and information.

\section{References}

1. Gelfand, I. M., Fuchs, D. B.: Cohomologies of the Lie algebra of vector fields on the circle. Funct. Anal. Appl. 2 (4), 92-93 (English translation 114-126) (1968)

2. Belavin, A. A., Polyakov, A. M., Zamolodchikov, A. B.: Infinite conformal symmetry in two-dimensional quantum field theory. Nucl. Phys. B241, 333-380 (1984)

3. Dotsenko, V. S., Fateev, V. A.: Conformal algebra and multipoint correlation functions in 2D statistical models. Nucl. Phys. B240, 312-348 (1984)

4. Belavin, A. A., Polyakov, A. M., Zamolodchikov, A. B.: Infinite conformal symmetry of critical fluctuations in two dimensions. J. Stat. Phys. 34 516, 763-774 (1984)

5. Friedan, D., Qiu, Z., Shenker, S.: Conformal invariance, unitarity and critical exponents in two dimensions. Phys. Rev. Lett. 52, 1575-1578 (1984)

6. Kac, V. G.: Highest weight representations of infinite-dimensional Lie algebras: Proceedings of the international congress of Mathematicians (Helsinki 1978). Acad. Sci. Fennica, Helsinki, 299-304 (1980)

7. Goddard, P., Olive, D.: Kac-Moody algebras, conformal symmetry and critical exponents. Nucl. Phys. B257, 83 (1985); Goddard, P., Kent, A., Olive, D.: Unitary representations of the Virasoro and super-Virasoro algebras. Commun. Math. Phys. 103, 105 (1986)

8. Friedan, D., Qiu, S., Shenker, S.: Details of the non-unitary proof for highest weight representations of the Virasoro algebra. Commun. Math. Phys. 107, 535-542 (1986)

9. Feigin, B. L., Fuchs, D. B.: Verma modules over the Virasoro algebra. Lecture Notes in Mathematics, vol. 1060, pp. 230-245. Berlin, Heidelberg, New York: Springer 1984

10. Kaplansky, I., Santharoubane, L. J.: Harish-Chandra modules over the Virasoro algebra. MSRI Publications vol. 5, pp. 217-231. Berlin, Heidelberg, New York: Springer 1985

11. Feigin, B. L., Fuchs, D. B.: Invariant skew-symmetric differential operators on the line and Verma modules over the Virasoro algebra. Funct. Anal. Appl. 16(2), 47-63; English translation $114-126$ (1982)

12. Kostrikin, I. A.: Irreducible graded representations of Lie algebras of Cartan type. Sov. Math. Dokl. 19, 1369-71 (1978)

13. Kac, V. G.: Some problems of infinite-dimensional Lie algebras and their representations. Lecture Notes in Mathematics, vol. 933, pp. 117-126. Berlin, Heidelberg, New York: Springer 1982

14. Chari, V., Pressley, A.: Unitary representations of the Virasoro algebra and a conjecture of Kac, Compositio Mathematica 67, 315-342 (1988)

15. Martin, C., Piard, A.: On a class of representations of the Virasoro algebra and a conjecture of Kac. Preprint 1990. Université de Bourgogne. Dijon, France

16. Mathieu, O.: Classification of Harish-Chandra modules over the Virasoro Lie algebra. Preprint 1990, I.A.S., Princeton

17. Whittaker, E. T., Watson, G. N.: A course of modern analysis, 4th edition. Cambridge: Cambridge University Press 1978 